\title{
STRONG IDENTIFIABILITY AND OPTIMAL MINIMAX RATES FOR FINITE MIXTURE ESTIMATION ${ }^{1}$
}

\author{
By PHILIPPE HEINRICH AND JONAS KAHN
}

\author{
Université Lille 1 and Université de Toulouse, CNRS
}

\begin{abstract}
We study the rates of estimation of finite mixing distributions, that is, the parameters of the mixture. We prove that under some regularity and strong identifiability conditions, around a given mixing distribution with $m_{0}$ components, the optimal local minimax rate of estimation of a mixing distribution with $m$ components is $n^{-1 /\left(4\left(m-m_{0}\right)+2\right)}$. This corrects a previous paper by Chen [Ann. Statist. 23 (1995) 221-233].

By contrast, it turns out that there are estimators with a (nonuniform) pointwise rate of estimation of $n^{-1 / 2}$ for all mixing distributions with a finite number of components.
\end{abstract}

1. Introduction. Finite mixture models have been applied since Pearson (1894) in various fields including astronomy, biology, genetics, economy, social sciences and engineering [McLachlan and Peel (2000)].

Finite mixtures and their estimation naturally arise mostly in three cases. One is model-based clustering. Here, the aim is to divide the data into $k$ clusters and assign (new) data to a cluster. A possible approach is to consider that data point from each cluster is generated according to a probability distribution, so that the whole data is generated by mixture with $k$ components [McLachlan and Peel (2000), Teh (2010)].

The second, more traditional case, is the statistical description of possibly heterogeneous data where the underlying mixing distribution has no particular meaning. In that case, mixtures are a tool to describe efficiently the "true" probability distribution and control the convergence rate of mixture estimators to it [van de Geer (1996), Ghosal and van der Vaart (2001), Genovese and Wasserman (2000)].

In the third case, the goal is the mixing distribution itself: its support points and proportions are the parameters we want to estimate. They typically correspond to the phenomenon that is studied. This is the case we are interested in, on the basis of observations drawn from the mixture.

Some works try to bridge the gap between the estimation of the mixture and the one of the mixing distribution, usually at least through estimation of the number of components - the order-in the finite mixture. In particular, Rousseau and

Received July 2015; revised October 2017.

${ }^{1}$ Supported in part by the Labex CEMPI (ANR-11-LABX-0007-01).

MSC2010 subject classifications. Primary 62G05; secondary 62G20.

Key words and phrases. Local asymptotic normality, convergence of experiments, maximum likelihood estimate, Wasserstein metric, mixing distribution, mixture model, rate of convergence, strong identifiability, pointwise rate, superefficiency. 
Mengersen (2011) have proved that their Bayesian estimator of an overfitted mixture tends to empty the extra components, and Gassiat and van Handel (2013) have given the minimal penalty on the maximum likelihood estimator of the order that yields strong consistency.

One could expect that a good estimator for the mixture would be a good estimator for the mixing model. However, this is not so clear. The situation is reminiscent of the difference between estimation and identification in model selection, where Yang (2005) has proved that no procedure can be optimal for both. Moreover, rates of convergence can be very different, as illustrated in an infinite-dimensional case by Bontemps and Gadat (2014).

Optimal rates are a key information in estimating the mixture parameters. These were unknown [see, e.g., Titterington, Smith and Makov (1985)] until the work of Chen (1995), who established a $n^{-1 / 4}$ local minimax rate, under reasonable identifiability conditions, for one-dimensional-parameter mixtures. This result is somewhat surprising since the rate does not depend on the number of components. It turns out to be erroneous, because of its Lemma 2.

Our article aims at giving correct statements and proofs and its consequences.

The main part consists in finding the correct exponent in the local minimax rate; that are Theorem 3.2 and Theorem 3.3. The rate gets worse with more components, which is consistent with the behaviour when there are infinitely many components, such as deconvolution: Fan (1991) had proved that the $L^{2}$-convergence rate was polylogarithmic in general, and Caillerie et al. (2013) and Dedecker and Michel (2013) have generalized this kind of rates to the more relevant (for us) Wasserstein metrics. The most original technical tool we shall use is a coarse-graining tree on the parameter indices.

In addition, the optimal local minimax rate and the optimal pointwise rate of estimation everywhere are not the same. This discrepancy is unusual in statistics, and probably the reason why the $n^{-1 / 4}$ rate went unchallenged for twenty years. Specifically, if instead of comparing all pairs of mixtures in a ball, we allow only one mixture in it, we get (21) which corrects Lemma 2 of Chen. As a consequence, Theorem 2 of Chen is valid by dropping uniformity: for any fixed mixing distribution say $G$, the estimator considered there will converge at rate $n^{-1 / 4}$, but with a multiplicative constant that depends on $G$. It then becomes a statement on the optimal pointwise rate of estimation everywhere, and can even be strengthened to $n^{-1 / 2}$ as we show in Theorem 4.1 .

The paper by Chen (1995) has been widely cited and used.

Apart from applied papers citing it that may have relied on the theoretical guarantees [see, e.g., Kuhn et al. (2014), Liu and Hancock (2014)], there are essentially two ways it could play a role. First, when it is used as part of a proof, secondly when it is used as a benchmark.

The first case covers papers that generalize Chen's result in other settings, and re-use its theorems and proofs. For example, Ishwaran, James and Sun (2001) propose a Bayesian estimator that achieves the $n^{-1 / 4}$ frequentist rate, and use Chen 
(1995), Lemma 2, in their analysis. More recently, Nguyen (2013) generalizes those results to mixtures with an abstract parameter space and indefinite number of components. But Nguyen (2013), Theorem 1, generalizes Chen (1995), Lemma 2, while transposing the proof with the mistake. The main results of both these articles hold; however, they do not need the full strength of Chen (1995), Lemma 2, but merely the weaker version (21). Ho and Nguyen (2016a) prove such a sufficient version.

These two papers also use Chen's (1995) article as a benchmark. However, the optimal pointwise rate everywhere would probably be a better reference point in their case, as in many others. In particular, it seems likely that a Bayesian estimator could converge pointwise at speed $n^{-1 / 2}$ everywhere. We have not checked whether the proof by Ishwaran, James and Sun (2001) can be improved, or if another prior is necessary.

This use as a benchmark is very usual, as expected for this kind of optimality result [see, e.g., Zhu and Zhang $(2006,2004)]$. Let us point in particular to a result by Martin (2012). He achieves almost $n^{-1 / 2}$ rate for the predictive recursion algorithm, and tries to explain the discrepancy with Chen (1995) by the fact that the parameters are constrained to live in a finite space for his algorithm. In fact, since his rate is pointwise, it fits with the continuous case.

In parallel with early available versions of this article, there have been interesting new developments: Ho and Nguyen (2015), Ho and Nguyen (2016b) have made explicit, among others, the system of equations underlying the minimax argument, for any finite number of parameters, and solved important special cases. The strong identifiability conditions we shall use in this article ensure that the system of equations is generic.

In Section 2, we give the notation and define and discuss the regularity assumptions we use. In Section 3, we state and discuss the main theorem, giving the optimal local minimax rate. In Section 4, pointwise rate everywhere is investigated. We try to give some intuition in both of these sections. In Section 5, we also dwell on the interpretation and practical consequences of having different rates, and conclude with open questions. In Section 6, we give and explain the meaning of the key intermediate results and prove the main theorems from here. In Section 7, we prove those key intermediate results. In particular, we introduce the most original tool of our proofs: the coarse-graining tree that allows to patch the mistake in the article by Chen (1995).

Some auxiliary and technical results are detailed in appendices grouped in a Supplementary Material [Heinrich and Kahn (2018)].

\section{Notation and regularity conditions.}

2.1. Basic notation. Throughout the paper, the parameter set $\Theta$, of diameter Diam $\Theta$, is always assumed to be a compact subset of $\mathbb{R}$ with nonempty interior. 
Let $\mathcal{G}_{m}$ (resp., $\mathcal{G}_{\leq m}$ ) be the set of (resp., at most) $m$-mixing or $m$-points support distributions $G$ on $\Theta$. We set also $\mathcal{G}_{<\infty}=\bigcup_{\ell>1} \mathcal{G}_{\ell}$.

As usual, we compare two mixing distributions $G$ and $G^{\prime}$ using transportation distances, or $L^{q}$-Wasserstein metrics, with $q \geq 1$. They completely bypass identifiability issues that would arise with the square error on parameters. The definition is

$$
W_{q}\left(G, G^{\prime}\right)=\inf _{\Pi}\left[\int_{\Theta^{2}}\left|\theta-\theta^{\prime}\right|^{q} \mathrm{~d} \Pi\left(\theta, \theta^{\prime}\right)\right]^{1 / q},
$$

where the infimum is taken over probability measures $\Pi$ on $\Theta \times \Theta$ with marginals $G$ and $G^{\prime}$. By Jensen's inequality, $W_{q} \geq W_{q^{\prime}}$ if $q>q^{\prime}$, and moreover, $W_{q}^{q} \leq$ $W_{q^{\prime}}^{q^{\prime}}(\text { Diam } \Theta)^{q-q^{\prime}}$. We will usually work with the strongest available Wasserstein metric for our results. Endowed with the metric $W_{q}$, the space $\mathcal{G}_{\leq m}$ is compact.

In the special case of $W_{1}$, we will also use its dual representation, where $|f|_{\text {Lip }}$ stands for the Lipschitz seminorm of $f$ [e.g., Dudley (2002), Section 11.8]:

$$
W_{1}\left(G, G^{\prime}\right)=\sup _{|f|_{\text {Lip }} \leq 1} \int_{\Theta} f(\theta) \mathrm{d}\left(G-G^{\prime}\right)(\theta) .
$$

Given $G=\sum_{j=1}^{m} \pi_{j} \delta_{\theta_{j}} \in \mathcal{G}_{m}$ and a family $\{f(x, \theta)\}_{\theta \in \Theta}$ of probability densities on $\mathbb{R}$ w.r.t. some $\sigma$-finite measure $\lambda$, a finite mixture model with $m$ components is defined by

$$
f(x, G)=\int_{\Theta} f(x, \theta) \mathrm{d} G(\theta)=\sum_{j=1}^{m} \pi_{j} f\left(x, \theta_{j}\right) .
$$

To compare mixture distribution functions $F(x, G)$ and $F\left(x, G^{\prime}\right)$, we will use the Kolmogorov metric $\left\|F(\cdot, G)-F\left(\cdot, G^{\prime}\right)\right\|_{\infty}$. Here, of course, we have by definition $F(x, \theta)=\int_{-\infty}^{x} f(y, \theta) \mathrm{d} \lambda(y)$, which extends to $F(x, G)$ by linearity providing that $f\left(y, \delta_{\theta}\right)=f(y, \theta)$ where $\delta_{\theta}$ denotes the Dirac measure at $\theta$.

2.2. Regularity conditions. ( $p, \alpha)$-smoothness. Hereafter, $f^{(p)}(x, \theta)$ or $f^{(p)}(\cdot, \theta)$ denote the $p$ th derivative of $f$ always taken w.r.t. the variable $\theta$.

Definition 2.1. The family $\{f(\cdot, \theta), \theta \in \Theta\}$ w.r.t. some $\sigma$-finite measure $\lambda$ is $(p, \alpha)$-smooth if

$$
E_{p, \alpha}\left(\theta, \theta^{\prime}, \theta^{\prime \prime}\right)=\int_{\mathbb{R}}\left|\frac{f^{(p)}\left(x, \theta^{\prime}\right)}{f\left(x, \theta^{\prime \prime}\right)}\right|^{\alpha} f(x, \theta) \mathrm{d} \lambda(x)
$$

is a well defined $[0, \infty]$-valued continuous function on $\Theta^{3}$, and if there exists $\varepsilon>0$ such that

$$
\left|\theta^{\prime}-\theta^{\prime \prime}\right|<\varepsilon \quad \Longrightarrow \quad \forall \theta \in \Theta, \quad E_{p, \alpha}\left(\theta, \theta^{\prime}, \theta^{\prime \prime}\right)<\infty .
$$


These smoothness conditions are easy to check in practice, and general enough. For example, all exponential families satisfy them, as shown in the Supplementary Material [Heinrich and Kahn (2018), E.2]. They will be useful for proving local asymptotic normality [Le Cam (1986)] of relevant families.

$k$-strong identifiability $(k \in \mathbb{N})$. Chen (1995) introduced a notion of strong identifiability. We will need a slightly more general version.

DEFINITION 2.2. The family $\{F(\cdot, \theta), \theta \in \Theta\}$ of distribution functions is $k$ strongly identifiable if for any finite set of say $m$ distinct points $\theta_{j} \in \Theta$,

$$
\left\|\sum_{p=0}^{k} \sum_{j=1}^{m} a_{p, j} F^{(p)}\left(\cdot, \theta_{j}\right)\right\|_{\infty}=0 \quad \Longrightarrow \quad\|a\|=\max _{p, j}\left|a_{p, j}\right|=0 .
$$

Of course, $\|\cdot\|_{\infty}$ denotes the supremum norm with respect to the variable $x$.

Chen's strong identifiability corresponds to 2-strong identifiability. Let us exemplify why this notion is useful. Consider $G_{n}=\frac{1}{2}\left(\delta_{n^{-1}}+\delta_{-n^{-1}}\right)$ in $\mathcal{G}_{2}$. We see that $F\left(\cdot, G_{n}\right)=F(\cdot, 0)+n^{-2} F^{(2)}(\cdot, 0) / 2+o\left(n^{-2}\right)$, provided we can expand around $\theta=0$. Then 2-strong identifiability ensures that $\left\|F(\cdot, 0)-F\left(\cdot, G_{n}\right)\right\|_{\infty}$ is of order $n^{-2}$, as shown in Proposition 2.3 below, whereas simple (1-strong) identifiability would say nothing. We will need $k$-strong identifiability with a higher $k$ if more cancellations of terms occur.

Proposition 2.3. Let $\{F(\cdot, \theta), \theta \in \Theta\}$ be $k$-strongly identifiable family of distribution functions with $F^{(k)}(x, \theta)$ continuous in $\theta$. Set for $\varepsilon>0$ :

$$
\Theta_{\varepsilon}^{m}=\left\{\left(\theta_{j}\right)_{1 \leq j \leq m} \subset \Theta: \min _{j \neq j^{\prime}}\left|\theta_{j}-\theta_{j^{\prime}}\right| \geq \varepsilon\right\} .
$$

Then, for all $a=\left(a_{p, j}\right)_{\substack{0 \leq p \leq k \\ 1 \leq j \leq m}}$,

$$
\inf _{\Theta_{\varepsilon}^{m}}\left\|\sum_{p=0}^{k} \sum_{j=1}^{m} a_{p, j} F^{(p)}\left(\cdot, \theta_{j}\right)\right\|_{\infty} \underset{\varepsilon, k, m}{\succcurlyeq}\|a\|,
$$

where $\underset{\varepsilon, k, m}{\succcurlyeq}$ means "more than", up to some constant $C(\varepsilon, k, m)>0$.

Proof. The function $\left(a,\left(\theta_{j}\right)_{1 \leq j \leq m}\right) \mapsto\left\|\sum_{p=0}^{k} \sum_{j=1}^{m} a_{p, j} F^{(p)}\left(\cdot, \theta_{j}\right)\right\|_{\infty}$ is lower semi-continuous on the compact set $\{a:\|a\|=1\} \times \Theta_{\varepsilon}^{m}$, so that it admits a minimum. By $k$-strong identifiability, it is nonzero.

We expect the strong identifiability to be rather generic, and hence the statements of this paper often meaningful. In particular, Chen (1995), Theorem 3, has proved that location and scale families with smooth densities are 2-strongly identifiable. The theorem and the proof straightforwardly generalize to our setting. We merely state the result. 
THEOREM 2.4. Let $k \geq 1$. Let $f$ be a probability density w.r.t. the Lebesgue measure on $\mathbb{R}$. Assume that $f$ is $k-1$ times differentiable with

$$
\lim _{x \rightarrow \pm \infty} f^{(p)}(x)=0 \quad \text { for } p \in \llbracket 0, k-1 \rrbracket .
$$

Consider $f(x, \theta)=f(x-\theta)$, with $\theta \in \Theta \subset \mathbb{R}$. Then the corresponding distributions family $\{F(\cdot, \theta), \theta \in \Theta\}$ is $k$-strongly identifiable. If $\Theta \subset(0, \infty)$, the result stays true with $f(x, \theta)=\frac{1}{\theta} f\left(\frac{x}{\theta}\right)$.

For more general conditions, see the article by Holzmann, Munk and Stratmann (2004), that also generalize well to $k$-strong identifiability.

3. Assumptions and main results on local asymptotic minimax rate. The statistical estimation will always be done in the model $\mathcal{G}_{\leq \mathbf{m}}$ and, for local statements, around a fixed mixture $G_{0} \in \mathcal{G}_{m_{0}}$ with $m_{0} \leq \mathbf{m}$. Set once and for all

$$
d_{0}=\mathbf{m}-m_{0} .
$$

Lower bounds on local asymptotic minimax rates.

Assumption $\mathrm{A}\left(k, \theta_{0}\right)$. For all $(p, \alpha) \in \llbracket 1,2 k+2 \rrbracket \times \llbracket 1,4 \rrbracket$, the family of densities $\{f(\cdot, \theta)\}_{\theta \in \Theta}$ is $(p, \alpha)$-smooth and satisfies, for some point $\theta_{0}$ in the interior of $\Theta$,

$$
\int\left|f^{(2 k+1)}\left(\cdot, \theta_{0}\right)\right| \mathrm{d} \lambda>0
$$

Typically, $k$ will be $d_{0}$ and $\theta_{0}$ a support point of $G_{0}$. These conditions allow to prove local asymptotic normality [Le Cam (1986)] for relevant families. This will give some insight on the reason why the lower bound on the rate holds, and on how the mixtures behave when we change the parameters in the least sensitive direction. The condition on the support point guarantees identifiability locally for the families, and we need more derivatives than usual, since there will be cancellations in the first terms.

REMARK 3.1. When comparing sequences, we will write $a_{n} \preccurlyeq b_{n}$ or $a_{n}=$ $O\left(b_{n}\right)$ for $a_{n} \leq C b_{n}$ where $C>0$ does not depend on $n$. We will furthermore use $a_{n} \asymp b_{n}$ for $b_{n} \preccurlyeq a_{n} \preccurlyeq b_{n}$. If needed, the dependence of $C$ on other parameters, say $u, \theta$ will be stressed by subscripts: $a_{n} \underset{u, \theta}{\preccurlyeq} b_{n}$ or $a_{n} \underset{u, \theta}{\asymp} b_{n}$.

In what follows, $\mathbb{E}_{G}$ will denote the expectation w.r.t. the measure $\mathrm{d} \mathbb{P}_{G}=$ $f(\cdot, G) \mathrm{d} \lambda$. And all the mixing distribution estimators denoted by $\widehat{G}_{n}$ below will be based on i.i.d. $n$-samples. 
THEOREM 3.2. Recall (6) and set $\varepsilon_{n}=n^{-1 /\left(4 d_{0}+2\right)+\kappa}$ for some $\kappa>0$. Let $\theta_{0}$ be a support point of $G_{0}$. Under Assumption $\mathrm{A}\left(d_{0}, \theta_{0}\right)$, for any sequence of estimators $\widehat{G}_{n}$, we have

$$
\sup _{\substack{G \in \mathcal{G}_{\mathbf{m}} \\ W_{1}\left(G, G_{0}\right)<\varepsilon_{n}}} \mathbb{E}_{G}\left[W_{1}\left(G, \widehat{G}_{n}\right)\right] \succcurlyeq n^{-1 /\left(4 d_{0}+2\right)} .
$$

The data we have access to is the empirical distribution $F_{n}$ where $n$ is the sample size, and which gets closer to the true mixture $F(\cdot, G)$ at rate $n^{-1 / 2}$. Hence two mixing distributions $G$ and $G^{\prime}$ can be told apart only if $\left\|F(\cdot, G)-F\left(\cdot, G^{\prime}\right)\right\|_{\infty}$ is at least of order $n^{-1 / 2}$.

As an example, let $G_{0}=\delta_{0}$ and consider two-component mixing distributions around, say $G_{n}=\frac{1}{2}\left(\delta_{-2 n^{-1 / 6}}+\delta_{2 n^{-1 / 6}}\right)$ and $G_{n}^{\prime}=\frac{4}{5} \delta_{-n^{-1 / 6}}+\frac{1}{5} \delta_{4 n^{-1 / 6}}$. Both have 0 as first moment, and $4 n^{-1 / 3}$ as second moment but the third moment is zero for $G_{n}$ and $12 n^{-1 / 2}$ for $G_{n}^{\prime}$. A Taylor expansion in $\theta=0$ up to the third order gives then $F\left(\cdot, G_{n}\right)-F\left(\cdot, G_{n}^{\prime}\right)=o\left(n^{-1 / 6}\right)$. So that no test can reliably tell $G_{n}$ from $G_{n}^{\prime}$ with an $n$-sample. On the other hand, we clearly have $W_{1}\left(G_{n}, G_{n}^{\prime}\right)=n^{-1 / 6}$ for all $n$. So that the minimax rate for two-mixing distributions cannot be better than $n^{-1 / 6}$.

This moment matching argument can be made rigorous and precise with two tools. One is Lindsay's Hankel trick [Lindsay (1989), Theorem 2A], also used by Dacunha-Castelle and Gassiat (1997) to estimate the order of a mixture. The other is local asymptotic normality property (LAN) developed by Le Cam (1986). Section 6 uses them to build a LAN family with scale factor $n^{1 /\left(4 d_{0}+2\right)}$ which gives Theorem 3.2 via Theorem 6.1.

Upper bounds on local asymptotic minimax rates.

Assumption $\mathrm{B}(k)$. The family of densities $\{f(\cdot, \theta)\}_{\theta \in \Theta}$ satisfies:

- For all $x, F(x, \theta)=\int_{-\infty}^{x} f(\cdot, \theta) \mathrm{d} \lambda$ is $k$-differentiable w.r.t. $\theta$,

- $\{F(\cdot, \theta), \theta \in \Theta\}$ is $k$-strongly identifiable,

- There is a uniform continuity modulus $\omega(\cdot)$ such that

$$
\sup _{x}\left|F^{(k)}(x, \theta)-F^{(k)}\left(x, \theta^{\prime}\right)\right| \leq \omega\left(\theta-\theta^{\prime}\right) \quad \text { with } \lim _{h \rightarrow 0} \omega(h)=0 .
$$

The latter condition holds if $\sup _{x, \theta}\left|F^{(k+1)}(x, \theta)\right|$ exists and is finite. These differentiability conditions should be compared with the usual parametric case, where differentiability in quadratic mean, or twice differentiability in $\theta$ for a less technical condition, is enough to get $n^{-1 / 2}$ local minimax rate. We will need $\mathrm{B}(2 \mathbf{m})$ to prove a global minimax rate of $n^{-1 /(4 \mathbf{m}-2)}$ [see (9) in Theorem 3.3], and B(1) for a pointwise rate of $n^{-1 / 2}$ everywhere (Theorem 4.1). 
THEOREM 3.3. Let $\widehat{G}_{n}(\mathbf{m})$ be "the" minimum distance estimator, that is any mixing distribution in $\mathcal{G}_{\leq \mathbf{m}}$ such that

$$
\left\|F\left(\cdot, \widehat{G}_{n}(\mathbf{m})\right)-F_{n}\right\|_{\infty}=\inf _{G \in \mathcal{G}_{\leq \mathbf{m}}}\left\|F(\cdot, G)-F_{n}\right\|_{\infty} .
$$

Under Assumption $\mathrm{B}(2 \mathbf{m})$, there is $\varepsilon>0$ such that, with $q=2 d_{0}+1$ :

$$
\sup _{\substack{G \in \mathcal{G}_{\leq \mathbf{m}} \\ W_{q}\left(G, G_{0}\right)<\varepsilon}} \mathbb{E}_{G}\left[W_{q}\left(\widehat{G}_{n}(\mathbf{m}), G\right)\right] \preccurlyeq n^{-1 /(2 q)},
$$

and more globally, with $r=2 \mathbf{m}-1$ :

$$
\sup _{G \in \mathcal{G}_{\leq \mathbf{m}}} \mathbb{E}_{G}\left[W_{r}\left(\widehat{G}_{n}(\mathbf{m}), G\right)\right] \preccurlyeq n^{-1 /(2 r)} .
$$

REMARK 3.4. Since $G \mapsto\left\|F(\cdot, G)-F_{n}\right\|_{\infty}$ is lower semi-continuous on the compact metric space $\left(\mathcal{G}_{\leq \mathbf{m}}, W_{q}\right)$, the infimum in (7) is attained. The minimum distance estimator is discussed by Deely and Kruse (1968) and Chen.

Theorem 3.3 is proved it by establishing a uniform control of the ratio $\left\|F(\cdot, G)-F\left(\cdot, G^{\prime}\right)\right\|_{\infty} / W_{q}\left(G, G^{\prime}\right)^{q}$ in Theorem 6.3. To do so, we consider sequences of couples $\left(G_{n}, G_{n}^{\prime}\right)$ minimizing the relevant ratios, and expand $F\left(\cdot, G_{n}\right)-F\left(\cdot, G_{n}^{\prime}\right)$ as a weighted sum on the relevant derivatives $F^{(p)}\left(\cdot, \theta_{j, n}\right)$. A difficulty arises since distinct support points $\theta_{j, n}$ may converge to the same $\theta_{j}$, leading to cancellations in the sums. Forgetting this case was the mistake in the proof of Chen (1995), Lemma 2. We overcome the issue in Section 7: we build clusters of support points whose pairwise distances decrease at a given rate and structured as nodes of a coarse-graining tree. We may then use Taylor expansions on each node and its descendants (Lemma 7.4).

REMARKS 3.5. It is worth to notice the following from Theorems 3.2-3.3:

- They together imply that the optimal local asymptotic minimax rate is $n^{-1 /\left(4 d_{0}+2\right)}$ for estimating a mixture with at most $\mathbf{m}$ components around a mixture with $m_{0}$ components, for any transportation distance $W_{p}$ with $p \in$ $\llbracket 1,2 d_{0}+1 \rrbracket$.

- The rate is driven by $d_{0}$, that is, it gets harder to estimate the parameters of a mixture when it is close to a mixture with less components.

- The worst case is when $m_{0}=1$, yielding a global minimax rate of estimation $n^{-1 /(4 \mathbf{m}-2)}$. The rate gets worse when more components are allowed. So that the nonparametric rates for estimating mixtures with an infinite number of components like in deconvolution appear natural.

- On the other hand, when the number of components is known, that is, $\mathbf{m}=m_{0}$, we have the usual local minimax rate $n^{-1 / 2}$.

- The global minimax rate on the mixtures with exactly $\mathbf{m}$ components stays at $n^{-1 /(4 \mathbf{m}-2)}$, because $\mathcal{G}_{\mathbf{m}}$ is not compact, and Theorem 3.2 still apply in the vicinity of $m_{0}$-component mixtures. 
4. On pointwise rate and superefficiency. The slow rate $n^{-1 /(4 \mathbf{m}-2)}$ in (9) might be a little surprising when, for example, some Bayesian estimators have $n^{-1 / 4}$ rate of convergence [Ishwaran, James and Sun (2001)]. However, this convergence rate is not the local minimax rate, but is closer to a pointwise rate of convergence, that is the speed at which an estimator converges to a fixed $G$ when $n$ increases. The difference with local minimax may be viewed as the loss of uniformity in $G$. We study here the optimal pointwise rates everywhere.

One motivation for local minimax results was to make clear how the Hodges' estimator [van der Vaart (1998), Chapter 8] and other superefficient estimators could cohabit with Craméer-Rao bound, and how much they could improve on it.

Specifically, a superefficient estimator can have a better pointwise convergence rate than any regular estimator, but not a better local minimax convergence rate [Hájek (1972)]. Moreover, it turns out that they can only have a better pointwise rate on a Lebesgue-null set [van der Vaart (1998), Chapter 8].

Now, the set of parameters (weights and support points) defining $\mathcal{G}_{<\mathbf{m}}$ is a Lebesgue-null w.r.t. the one defining $\mathcal{G}_{\leq \mathbf{m}}$. Hence, we might expect that, by biasing the estimators toward the low numbers of components, we might attain better pointwise rates on $\mathcal{G}_{<\mathbf{m}}$, up to $n^{-1 / 2}$, which is the value when the number of components is known. By letting $\mathbf{m}$ go to infinity, we would have this pointwise rate for all finite mixing distributions. It turns out this is indeed the case.

THEOREM 4.1. Consider for each $m \geq 1$ the minimum distance estimator $\widehat{G}_{n}(m)$ in $\mathcal{G}_{\leq m}$ as defined in Theorem 3.3, with $\widehat{G}_{n}(\infty)$ arbitrary. Fix $\kappa \in(0,1 / 2)$ and set

$$
\widehat{m}_{n}=\min \left\{m \geq 1:\left\|F\left(\cdot, \widehat{G}_{n}(m)\right)-F_{n}\right\|_{\infty} \leq n^{-1 / 2+\kappa}\right\} .
$$

Under Assumption $\mathrm{B}(1)$, for any finite mixing distribution $G \in \mathcal{G}_{<\infty}$,

$$
\mathbb{E}_{G}\left[W_{1}\left(\widehat{G}_{n}\left(\widehat{m}_{n}\right), G\right)\right] \underset{G, \kappa}{\preccurlyeq} n^{-1 / 2} .
$$

REMARKS 4.2. - Since the typical distance between empirical and theoretical distribution functions is $n^{-1 / 2}$, this $\widehat{m}_{n}$ in (10) is the lowest number of components that is not clearly insufficient.

- The rate $n^{-1 / 2}$ cannot be improved since it is the rate if the number of components is known beforehand.

- This is slightly stronger than just checking that we find the right number of components and then applying Theorem 3.3, because we need much less regularity. Only Assumption $\mathrm{B}(1)$ is required, instead of $\mathrm{B}(2 \mathrm{~m})$. That is, we do not need more smoothness when the number of components increases. Under the hood, we rely on bound (20) instead of bound (19) in Theorem 6.3.

- There are easy methods, conceptually and numerically, to reach the pointwise $n^{-1 / 2}$ rate. Estimate the mixture for each number of components $m$, and choose 
the first $m$ that is not clearly bad. We have used the minimum distance estimator for consistency within the article. But any method with a global $n^{-1 / 2}$ rate of convergence when the number of components is known will work in the same way, in particular, MLE. The important part is the model selection step (10). Other good model selection methods or Bayesian priors would also work.

5. Practical consequences and perspectives. Local minimax rate and pointwise rate everywhere disagreement might be rare enough that it is worth recalling first what it means.

The asymptotic rate of convergence to a given $G$ will be the pointwise rate $C_{G} n^{-1 / 2}$ where $C_{G}$ is some positive constant. However, the estimator will enter this asymptotic regime only after a long time. More precisely, it enters this regime after that $G$ is not anymore in any of the balls used in the local minimax bound. Alternatively, we may view this situation as the constant $C_{G}$ exploding when $G$ is close to certain $G_{0}$.

In our case, imagine we have a mixing distribution with three components, with all support points within distance $\delta>0$ of some $\theta_{0}$. Then about $\delta^{-(4(3-1)+2)}=$ $\delta^{-10}$ observations are necessary to get an estimator with an error of $\delta$. In particular, if $G$ and $G^{\prime}$ are two such three-component mixing distributions, chosen to have the same first four moments, and $\tilde{G}$ and $\tilde{G}^{\prime}$ are the same mixing distributions, rescaled to be ten times closer, we will need $10^{10}$ as many data points to tell them apart as for $G$ and $G^{\prime}$.

As a consequence, if the components of the mixing distribution to be estimated are not far apart one from the other, it is quite often impossible to get enough data points to get an appropriate estimate. An experimentalist with any leeway in what he measures (use of different markers, say) might then wish to ensure that the peaks are far apart, even at the cost of many data points.

We end the section by some thoughts on possible further work. This article contains the proof that the optimal local minimax rate of estimation around a mixing distribution with $m_{0}$ components among mixing distributions with $\mathbf{m}$ components is $n^{-1 /\left(4\left(\mathbf{m}-m_{0}\right)+2\right)}$, when the parameter space $\Theta$ is a compact subset of $\mathbb{R}$.

We think that extension to a multivariate $\Theta$ should be workable, much like Nguyen (2013) did for the former erroneous result. On the other hand, noncompactness of $\Theta$ would probably bring about technical difficulties, and cases where the result would not hold. Stronger forms of identifiability would probably be required in general, to avoid problems with limits. Moreover, for many natural higher-dimensional families, strong identifiability does not hold, so that the results would be different.

Finally, another line of inquiry are the results that might be expected in a Bayesian framework. The most natural equivalent to the convergence rate of the $a$ posteriori distribution to the real parameter is the pointwise rate of convergence. Hence the question: can we build Bayesian estimators where the a posteriori distributions converge at rate $n^{-1 / 2}$ everywhere? Of course, the convergence would not be uniform. 


\section{Key tools and proofs.}

6.1. Local asymptotic normality for Theorem 3.2. All the densities considered in the sequel are w.r.t. some given dominating $\sigma$-finite measure on $\mathbb{R}$. We call experiment a family $\mathcal{E}$ of densities.

THEOREM 6.1. Let $G_{0} \in \mathcal{G}_{m_{0}}$ with a support point $\theta_{0}$ in the interior of $\Theta$. There is a family $\left\{G_{n}(u)\right\}_{n \geq 0, u \in \mathbb{R}}$ in $\mathcal{G}_{\mathbf{m}}$ with the following properties:

(a) For all distinct $u, u^{\prime}$ in $\mathbb{R}$, we have together

$$
W_{1}\left(G_{n}(u), G_{n}\left(u^{\prime}\right)\right) \underset{u, u^{\prime}}{\succcurlyeq} n^{-1 /\left(4 d_{0}+2\right)} \succcurlyeq W_{1}\left(G_{n}(u), G_{0}\right) .
$$

(b) Assume $\mathrm{A}\left(d_{0}, \theta_{0}\right)$ for the family $\{f(\cdot, \theta)\}_{\theta \in \Theta}$ and set the product density $f_{n, u}=\bigotimes_{i=1}^{n} f\left(\cdot, G_{n}(u)\right)$. There is an increasing real sequence $U_{n} \rightarrow \infty$ such that the sequence of experiments $\mathcal{E}_{n}=\left(f_{n, u}\right)_{u \in\left[-U_{n}, U_{n}\right]}$ is locally asymptotically normal $(L A N)$ : there are random variables $Z_{n}$, asymptotically $\mathcal{N}(0,1)$, and numbers $\Gamma_{n}>0$ such that for all $u \in \mathbb{R}$,

$$
\log \left(\frac{f_{n, u}(X)}{f_{n, 0}(X)}\right)-u Z_{n} \sqrt{\Gamma_{n}}+\frac{u^{2}}{2} \Gamma_{n} \underset{n \rightarrow \infty}{\stackrel{P}{\longrightarrow}} 0,
$$

where $X$ is a n-sample of density $f_{n, 0}$.

In addition, we have $\liminf _{n} \Gamma_{n}>0$ and $\lim \sup _{n} \Gamma_{n}<\infty$.

REMARK 6.2. We want only an example of this slow convergence, and it should be somewhat typical. That is why we have chosen the regularity conditions to make the proof easy, while still being easy to check, in particular, for exponential families.

In particular, in Assumption $\mathrm{A}\left(d_{0}, \theta_{0}\right)$, it could probably be possible to lower $\alpha$ in $(p, \alpha)$-smoothness to $2+\varepsilon$ and still get the uniform bound we use in the law of large numbers below. Similarly, less differentiability might be necessary if we tried to imitate differentiability in quadratic mean.

Conversely, under possibly more stringent regularity conditions, $\Gamma_{n}$ is expected to converge to $\mathbb{E}_{G_{0}}\left|\frac{f^{\left(2 d_{0}+1\right)}\left(\cdot, \theta_{0}\right)}{f\left(\cdot, G_{0}\right)}\right|^{2}$ up to a multiplicative constant.

Proof OF THEOREM 6.1. Write the mixing distribution $G_{0}$ as

$$
G_{0}=\sum_{j=1}^{m_{0}-1} \pi_{j} \delta_{\theta_{j}}+\pi_{0} \delta_{\theta_{0}}
$$

with $\theta_{0}$ in the interior of $\Theta$. Let $u \in \mathbb{R}$ and replace the Dirac measure $\delta_{\theta_{0}}$ in (12) with a mixing distribution $H_{n}(u)$ :

$$
G_{n}(u)=\sum_{j=1}^{m_{0}-1} \pi_{j} \delta_{\theta_{j}}+\pi_{0} H_{n}(u) .
$$


We want to choose $H_{n}(u)$ close to $\delta_{\theta_{0}}$. To this end, set $\mu_{0}=1$ and $\mu_{2 d-1}=u$ with $d=d_{0}+1$. Choose in addition numbers $\mu_{1}, \ldots, \mu_{2 d-2}$ such that the $k \times k$ Hankel matrices $\left(M_{k}\right)_{i, j}=\mu_{i+j-2}$ satisfy $\operatorname{det} M_{k}>0$ for $k \in \llbracket 1, d-1 \rrbracket$. Then, by Lindsay's Theorem 2A (1989), there is a unique mixing distribution $H(u)=$ $\sum_{j=m_{0}}^{\mathbf{m}} \pi_{j}(u) \delta_{h_{j}(u)}$ with exactly $d$ support-points $h_{j}(u)$ and first moments $\mu_{k}$ up to order $2 d-1$ satisfying

$$
\sum_{j=m_{0}}^{\mathbf{m}} \pi_{j}(u) h_{j}(u)^{k}=\mu_{k}, \quad k \in \llbracket 0,2 d-1 \rrbracket .
$$

Define then $H_{n}(u)$ by shifting and rescaling the support points of $H(u)$ :

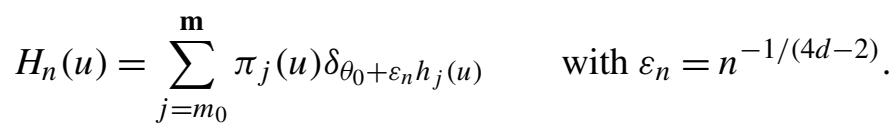

Now, using the dual representation (1) of $W_{1}$, we see that

$$
W_{1}\left(G_{n}(u), G_{0}\right)=\pi_{0} W_{1}\left(H_{n}(u), \delta_{\theta_{0}}\right)=\pi_{0} \varepsilon_{n} W_{1}\left(H(u), \delta_{0}\right)
$$

and likewise, $W_{1}\left(G_{n}(u), G_{n}\left(u^{\prime}\right)\right)$ equals $\pi_{0} \varepsilon_{n} W_{1}\left(H(u), H\left(u^{\prime}\right)\right)$ so that Theorem 6.1(a) follows.

To guarantee that the points $\theta_{0}+\varepsilon_{n} h_{j}(u)$ involved in $G_{n}(u)$ stay inside $\Theta$ uniformly in $u$, let us show that the functions $h_{j}(\cdot)$ are continuous. Consider the map

$$
\varphi\left(\pi_{1}, \ldots, \pi_{d}, h_{1}, \ldots, h_{d}\right)=\left(\sum_{1}^{d} \pi_{j}, \sum_{1}^{d} \pi_{j} h_{j}, \sum_{1}^{d} \pi_{j} h_{j}^{2}, \ldots, \sum_{1}^{d} \pi_{j} h_{j}^{2 d-1}\right)
$$

on the set $\left\{\left(\pi_{1}, \ldots, \pi_{d}, h_{1}, \ldots, h_{d}\right): \pi_{1}>0, \ldots, \pi_{d}>0, h_{1}<\cdots<h_{d}\right\}$. The uniqueness in Theorem 2A by Lindsay (1989) implies that $\varphi$ is injective. Moreover, its Jacobian is nonzero, as it can be seen by recurrence on $d$ :

$$
J(\varphi)=(-1)^{\frac{(d-1) d}{2}} \pi_{1} \cdots \pi_{d} \prod_{1 \leq j<k \leq d}\left(h_{j}-h_{k}\right)^{4} .
$$

Thus the inverse of $\varphi$ is locally continuous, so that, in particular, the $h_{j}(u)$ are all continuous.

Set now

$$
\mathbf{h}(U)=\max _{j \leq d} \max _{|u| \leq U}\left|h_{j}(u)\right|,
$$

which is finite for any $U>0$ and choose a positive sequence $\left(U_{n}\right)$ such that

$$
U_{n} \rightarrow \infty \text { and } \varepsilon_{n} \mathbf{h}\left(U_{n}\right) \rightarrow 0 .
$$

We can now prove local asymptotic normality (11). Let $X=\left(X_{1, n}, \ldots, X_{n, n}\right)$ be an i.i.d. sample with density $f_{n, 0}$. Since we proceed along the lines of Chen (1995), the proof is only sketched here. Write the log-likelihood ratio as

$$
\log \left(\frac{f_{n, u}(X)}{f_{n, 0}(X)}\right)=\sum_{i=1}^{n} \log \left(1+Y_{i, n}(u)\right)
$$


with

$$
Y_{i, n}(u)=\frac{f\left(X_{i, n}, G_{n}(u)\right)-f\left(X_{i, n}, G_{n}(0)\right)}{f\left(X_{i, n}, G_{n}(0)\right)} .
$$

The main steps are as follows [see Heinrich and Kahn (2018), Sections A.1, A.2 and A.3] for the details:

Step 1. Use linearity of $G \mapsto f(\cdot, G)$ and Taylor expansions up to the order $2 d-1$ with remainder on $Y_{i, n}(u)$ at $\theta_{0}$ to show that the r.v.'s

$$
Z_{i, n}=\pi_{0} \frac{f^{(2 d-1)}\left(X_{i, n}, \theta_{0}\right)}{f\left(X_{i, n}, G_{n}(0)\right)}
$$

are centered under $f_{n, 0}$.

Step 2. Define $\Gamma_{n}=\mathbb{E}_{G_{n}(0)}\left|Z_{1, n}\right|^{2}$ and $Z_{n}=n^{-1 / 2} \Gamma_{n}^{-1 / 2} \sum_{i=1}^{n} Z_{i, n}$ and prove that $Z_{n}$ is asymptotically $\mathcal{N}(0,1)$ via Lyapunov theorem for triangular arrays.

Step 3. Show the following convergences for all $u$ :

$$
\begin{aligned}
A_{n}(u) & :=\sum_{i=1}^{n} Y_{i, n}(u)-u Z_{n} \sqrt{\Gamma_{n}} \stackrel{L^{2}}{\rightarrow} 0, \\
B_{n}(u) & :=\sum_{i=1}^{n} Y_{i, n}(u)^{2}-u^{2} \Gamma_{n} \stackrel{L^{1}}{\rightarrow} 0, \\
C_{n}(u) & :=\sum_{i=1}^{n}\left|Y_{i, n}(u)\right|^{3} \stackrel{L^{1}}{\rightarrow} 0 .
\end{aligned}
$$

Then derive the LAN property from the equality

$$
\log \left(\frac{f_{n, u}(X)}{f_{n, 0}(X)}\right)-u Z_{n} \sqrt{\Gamma_{n}}+\frac{u^{2}}{2} \Gamma_{n}=A_{n}(u)+\frac{1}{2} B_{n}(u)+O_{P}\left(C_{n}(u)\right) .
$$

6.2. Proof of Theorem 3.2. Let us show how Theorem 6.1 entails Theorem 3.2 using just two points and contiguity [Le Cam (1960)]. Consider any sequence of estimators $\widehat{G}_{n}$, and $G_{n}(u)$ for $u=0,1$ as defined in Theorem 6.1. It is enough to show that for large $n$,

$$
\sup _{G \in\left\{G_{n}(0), G_{n}(1)\right\}} \mathbb{E}_{G}\left[W_{1}\left(G, \widehat{G}_{n}\right)\right] \succcurlyeq n^{-1 /\left(4 d_{0}+2\right)} .
$$

Recall that we set here $\varepsilon_{n}=n^{-1 /\left(4 d_{0}+2\right)+\kappa}$ and note that $G_{n}(0)$ and $G_{n}(1)$ are in the ball $\left\{G: W_{1}\left(G, G_{0}\right)<\varepsilon_{n}\right\}$ for large $n$, by Theorem $6.1(\mathrm{a})$.

Consider the probability measures $P_{n, u}$ with the densities $f_{n, u}$ of Theorem 6.1(b) for $u=0,1$ and set $g_{n}=\frac{f_{n, 1}(X)}{f_{n, 0}(X)} \exp \left(-Z_{n} \sqrt{\Gamma_{n}}+\Gamma_{n} / 2\right)$. Then

$$
P_{n, 1}(A)=\mathrm{e}^{-\frac{\Gamma_{n}}{2}} \int_{A} \mathrm{e}^{Z_{n} \sqrt{\Gamma_{n}}} g_{n} \mathrm{~d} P_{n, 0} \geq \frac{\mathrm{e}^{-\frac{\Gamma_{n}}{2}}}{2} \int_{A \cap\left\{Z_{n}>0\right\} \cap\left\{g_{n}>1 / 2\right\}} \mathrm{d} P_{n, 0} .
$$


We have $g_{n} \stackrel{P_{n, 0}}{\longrightarrow} 1$ by $(11)$ so that $P_{n, 0}\left(g_{n} \leq 1 / 2\right) \leq 1 / 16$ for large $n$. Since $Z_{n}$ is asymptotically $\mathcal{N}(0,1)$, we also have $P_{n, 0}\left(Z_{n} \leq 0\right) \leq 1 / 2+1 / 16$. Thus $P_{n, 0}\left(\left\{Z_{n}>0\right\} \cap\left\{g_{n}>1 / 2\right\}\right)$ is at least $3 / 8$ for $n$ large enough and

$$
P_{n, 0}(A) \geq \frac{3}{4} \quad \Longrightarrow \quad P_{n, 1}(A) \succcurlyeq \mathrm{e}^{-\Gamma_{n} / 2} .
$$

Now, choose $A=\left\{W_{1}\left(G_{n}(1), \widehat{G}_{n}\right) \geq a n^{-1 /\left(4 d_{0}+2\right)}\right\}$ where $a>0$ is such that $W_{1}\left(G_{n}(1), G_{n}(0)\right) \geq 2 a n^{-1 /\left(4 d_{0}+2\right)}$, by Theorem 6.1(a). By the triangle's inequality, the complement $A^{c}$ is included in $\left\{W_{1}\left(G_{n}(0), \widehat{G}_{n}\right) \geq a n^{-1 /\left(4 d_{0}+2\right)}\right\}$. Now, either we have $P_{n, 0}\left(A^{c}\right) \geq \frac{1}{4}$ and, for $G=G_{n}(0)$, we get

$$
\mathbb{E}_{G}\left[W_{1}\left(G, \widehat{G}_{n}\right) \mathbf{1}_{A^{c}}\right] \geq \frac{a}{4} n^{-1 /\left(4 d_{0}+2\right)},
$$

or we have $P_{n, 0}(A) \geq \frac{3}{4}$ and by using (17), for $G=G_{n}(1)$, we get

$$
\mathbb{E}_{G}\left[W_{1}\left(G, \widehat{G}_{n}\right) \mathbf{1}_{A}\right] \succcurlyeq \mathrm{e}^{-\Gamma_{n} / 2} a n^{-1 /\left(4 d_{0}+2\right)},
$$

so that (16) is proved since $\lim \sup _{n} \Gamma_{n}<\infty$.

6.3. Comparison between distances for Theorem 3.3 and Theorem 4.1. The key technical tool is the following.

THEOREM 6.3. - Let $G_{0} \in \mathcal{G}_{m_{0}}$. Under Assumption $\mathrm{B}(2 \mathbf{m})$, there are $\varepsilon>0$ and $\delta>0$ such that, with $q=2 d_{0}+1$ :

$$
\inf _{\substack{G \neq G^{\prime} \in \mathcal{G}_{\leq} \mathbf{m} \\ W_{q}\left(G, G_{0}\right)<\varepsilon \\ W_{q}\left(G^{\prime}, G_{0}\right)<\varepsilon}} \frac{\left\|F(\cdot, G)-F\left(\cdot, G^{\prime}\right)\right\|_{\infty}}{W_{q}\left(G, G^{\prime}\right)^{q}}>\delta,
$$

and more globally, with $r=2 \mathbf{m}-1$ :

$$
\inf _{G \neq G^{\prime} \in \mathcal{G}_{\leq \mathbf{m}}} \frac{\left\|F(\cdot, G)-F\left(\cdot, G^{\prime}\right)\right\|_{\infty}}{W_{r}\left(G, G^{\prime}\right)^{r}}>\delta .
$$

- Let $G_{0} \in \mathcal{G}_{m_{0}}$. Under Assumption $\mathrm{B}(1)$, there are $\varepsilon>0$ and $\delta>0$ such that

$$
\begin{aligned}
& \inf _{\substack{G \neq G^{\prime} \in \mathcal{G}_{\leq m_{0}} \\
W_{1}\left(G, G_{0}\right)<\varepsilon \\
W_{1}\left(G^{\prime}, G_{0}\right)<\varepsilon}} \frac{\left\|F(\cdot, G)-F\left(\cdot, G^{\prime}\right)\right\|_{\infty}}{W_{1}\left(G, G^{\prime}\right)}>\delta .
\end{aligned}
$$

- Let now $G_{0} \in \mathcal{G}_{\leq m_{0}}$. Under Assumption $\mathrm{B}(2)$, there are $\varepsilon>0$ and $\delta>0$ such that

$$
\inf _{\substack{G \in \mathcal{G}_{\leq m_{0}} \\ W_{1}\left(G, G_{0}\right)<\varepsilon}} \frac{\left\|F(\cdot, G)-F\left(\cdot, G_{0}\right)\right\|_{\infty}}{W_{1}\left(G, G_{0}\right)^{2}}>\delta .
$$


The proof of Theorem 6.3 is postponed to Section 7 where the novel ingredient, a coarse-graining tree, is constructed to prove (18) and (19), the most difficult points. These one entail Theorem 3.3. The two related bounds (20) and (21) hold under weaker differentiability assumptions, but are less general. Bound (20) covers the case where the number of components in the mixture is known, and is used for the proof of Theorem 4.1. Bound (21) is the valid weaker version of Lemma 2 by Chen (1995), which is sufficient for the use other authors have made of it. Here, we only compare mixtures in a ball with the mixture at the center of the ball.

For the proofs of Theorem 3.3 and Theorem 4.1, we need in addition the following.

LEMMA 6.4. Let $q, d \geq 1$ and $G \in \mathcal{G}_{\leq m}$. Assume that the minimum distance estimators $\widehat{G}_{n}:=\widehat{G}_{n}(m)$ defined in Theorem 3.3 satisfy for some constant $C>0$ and on some event $A$,

$$
W_{q}\left(\widehat{G}_{n}, G\right)^{d} \leq C\left\|F\left(\cdot, \widehat{G}_{n}\right)-F(\cdot, G)\right\|_{\infty} .
$$

Then

$$
\mathbb{E}_{G}\left[W_{q}\left(\widehat{G}_{n}, G\right)\right] \leq\left(2 \pi C^{2}\right)^{1 / 2 d} n^{-1 / 2 d}+\operatorname{Diam}(\Theta) \mathbb{P}_{G}\left(A^{c}\right) .
$$

Moreover, $\mathbb{P}_{G}\left(A^{c}\right)$ is at most $2 \mathrm{e}^{-2 n z^{2}}$ if $A$ is either $\left\{\left\|F_{n}-F(\cdot, G)\right\|_{\infty} \leq z\right\}$ or $\left\{\left\|F\left(\cdot, \widehat{G}_{n}\right)-F(\cdot, G)\right\|_{\infty} \leq 2 z\right\}$.

SKETCH OF PROOF. Bound $W_{q}\left(\widehat{G}_{n}, G\right)$ by $\operatorname{Diam}(\Theta)$ on $A^{c}$, use the definition (7) and the triangle's inequality to bound $\left\|F\left(\cdot, \widehat{G}_{n}\right)-F(\cdot, G)\right\|_{\infty}$ by $2 \| F_{n}-$ $F(\cdot, G) \|_{\infty}$, then use Jensen's inequality on $A$ and bound $\mathbb{E}_{G_{1}}\left\|F_{n}-F(\cdot, G)\right\|_{\infty}$ [and $\mathbb{P}_{G}\left(A^{c}\right)$ ] by applying DKW's inequality [Massart (1990)].

6.4. Proof of Theorem 3.3. Let $\varepsilon, \delta>0$ such (18) holds. Set

$$
z=\frac{1}{2} \inf _{\substack{G, G^{\prime} \in \mathcal{G}_{\leq \mathbf{m}} \\ W_{q}\left(G, G_{0}\right) \leq \varepsilon / 2 \\ W_{q}\left(G^{\prime}, G_{0}\right) \geq \varepsilon}}\left\|F(\cdot, G)-F\left(\cdot, G^{\prime}\right)\right\|_{\infty} .
$$

The infimum is taken over a compact set and is thus attained. We have $z>0$ by identifiability [coming from Assumption $\mathrm{B}(2 \mathbf{m})$ ].

Consider $A=\left\{\left\|F(\cdot, G)-F\left(\cdot, \widehat{G}_{n}(\mathbf{m})\right)\right\|_{\infty} \leq z\right\}$. If $G$ is in $\mathcal{G}_{\leq \mathbf{m}}$ with $W_{q}(G$, $\left.G_{0}\right) \leq \varepsilon / 2$, then $\widehat{G}_{n}(\mathbf{m})$ must satisfy $W_{q}\left(\widehat{G}_{n}(\mathbf{m}), G_{0}\right)<\varepsilon$ on the event $A$ so that by $(18)$,

On $A, \quad W_{q}\left(\widehat{G}_{n}(\mathbf{m}), G\right)^{q}<\frac{1}{\delta}\left\|F\left(\cdot, \widehat{G}_{n}(\mathbf{m})\right)-F(\cdot, G)\right\|_{\infty}$.

Applying Lemma 6.4 with $d=q=2 d_{0}+1$ and $C=1 / \delta$ yields

$$
\mathbb{E}_{G}\left[W_{q}\left(\widehat{G}_{n}(\mathbf{m}), G\right)\right] \leq\left(\frac{2 \pi}{\delta^{2}}\right)^{1 / 2 q} n^{-1 / 2 q}+2 \operatorname{Diam}(\Theta) \mathrm{e}^{-n z^{2} / 2}
$$

so that bound (8) is proved. Bound (9) is obtained likewise from (19). 
6.5. Proof of Theorem 4.1. Consider a mixing distribution $G_{0} \in \mathcal{G}_{m_{0}}$. Under Assumption $\mathrm{B}(1)$, let $\varepsilon, \delta>0$ such (20) holds. Fix $\kappa \in\left(0, \frac{1}{2}\right)$ and set

$$
z=n^{-1 / 2+\kappa} \wedge \frac{1}{4} \inf _{\substack{G \in \mathcal{G}_{\leq m_{0}} \\ W_{1}\left(G, G_{0}\right) \geq \varepsilon}}\left\|F(\cdot, G)-F\left(\cdot, G_{0}\right)\right\|_{\infty} .
$$

By compactness and identifiability, the infimum in $z$ is attained and positive. On the event $A=\left\{\left\|F\left(\cdot, G_{0}\right)-F_{n}\right\|_{\infty} \leq z\right\}$, the minimum distance estimator $\widehat{G}_{n}\left(m_{0}\right)$ in $\mathcal{G}_{\leq m_{0}}$, defined in Theorem 3.3, satisfies

$$
\left\|F\left(\cdot, \widehat{G}_{n}\left(m_{0}\right)\right)-F_{n}\right\|_{\infty} \leq\left\|F\left(\cdot, G_{0}\right)-F_{n}\right\|_{\infty} \leq z \leq n^{-1 / 2+\kappa}
$$

so that $\hat{m}_{n}$ is at most $m_{0}$ by (10); thus we have $\widehat{G}_{n}\left(\hat{m}_{n}\right) \in \mathcal{G}_{\leq m_{0}}$. Next, $\widehat{G}_{n}\left(\hat{m}_{n}\right)$ must satisfy $W_{1}\left(\widehat{G}_{n}\left(\hat{m}_{n}\right), G_{0}\right)<\varepsilon$ on $A$ since

$$
\left\|F\left(\cdot, \widehat{G}_{n}\left(\hat{m}_{n}\right)\right)-F\left(\cdot, G_{0}\right)\right\|_{\infty} \leq 2\left\|F\left(\cdot, G_{0}\right)-F_{n}\right\|_{\infty} \leq 2 z,
$$

by the triangle's inequality. Applying then (20) on $A$, we get

$$
W_{1}\left(\widehat{G}_{n}\left(\hat{m}_{n}\right), G_{0}\right)<\frac{1}{\delta}\left\|F\left(\cdot, \widehat{G}_{n}\left(\hat{m}_{n}\right)\right)-F\left(\cdot, G_{0}\right)\right\|_{\infty} \cdot
$$

Now, apply Lemma 6.4 with $q=d=1, C=1 / \delta$ and $A$ as above, so that

$$
\mathbb{E}_{G_{0}}\left[W_{1}\left(\widehat{G}_{n}\left(\hat{m}_{n}\right), G_{0}\right)\right] \leq \sqrt{\frac{2 \pi}{\delta}} n^{-1 / 2}+2 \operatorname{Diam}(\Theta) \exp \left(-2 n^{2 \kappa}\right) .
$$

\section{The coarse-graining tree and the proof of Theorem 6.3.}

7.1. Proof of (18): The coarse graining tree. Let $G_{0} \in \mathcal{G}_{m_{0}}$. We have to show that, under Assumption $\mathrm{B}(2 \mathbf{m})$, there is $\varepsilon>0$ such that

$$
L:=\inf _{\substack{G \neq G^{\prime} \in \mathcal{G}_{\leq \mathbf{m}} \\ W_{q}\left(G, G_{0}\right)<\varepsilon \\ W_{q}\left(G^{\prime}, G_{0}\right)<\varepsilon}} \frac{\left\|F(\cdot, G)-F\left(\cdot, G^{\prime}\right)\right\|_{\infty}}{W_{q}\left(G, G^{\prime}\right)^{q}}>0 \quad \text { with } q=2 d_{0}+1 .
$$

Assume on the contrary that $L=0$ and choose mixing distributions $G_{n}$ and $G_{n}^{\prime}$ in $\mathcal{G}_{\leq \mathbf{m}}$ with $W_{q}\left(G_{n}, G_{0}\right) \vee W_{q}\left(G_{n}^{\prime}, G_{0}\right)<1 / n$ such that for each $n \geq 1$, the ratios $\left\|\bar{F}\left(\cdot, G_{n}\right)-F\left(\cdot, G_{n}^{\prime}\right)\right\|_{\infty} / W_{q}\left(G_{n}, G_{n}^{\prime}\right)^{q}$ are less than $1 / n$. We shall prove, up to selecting subsequences, the following contradiction:

$$
\left\|F\left(\cdot, G_{n}\right)-F\left(\cdot, G_{n}^{\prime}\right)\right\|_{\infty} \succcurlyeq W_{q}\left(G_{n}, G_{n}^{\prime}\right)^{q} .
$$

Some notation. We may and do assume that there are integers $m, m^{\prime}$ at most $\mathbf{m}$ such that $\left(G_{n}\right) \subset \mathcal{G}_{m}$ and $\left(G_{n}^{\prime}\right) \subset \mathcal{G}_{m^{\prime}}$. We can then write $G_{n}=\sum_{j=1}^{m} \pi_{j, n} \delta_{\theta_{j, n}}$ and $G_{n}^{\prime}=\sum_{j=1}^{m^{\prime}} \pi_{j, n}^{\prime} \delta_{\theta_{j, n}^{\prime}}$ and set

$$
\left(\varpi_{j, n}, \vartheta_{j, n}\right)= \begin{cases}\left(\pi_{j, n}, \theta_{j, n}\right) & \text { if } j \leq m, \\ \left(-\pi_{j-m, n}^{\prime}, \theta_{j-m, n}^{\prime}\right) & \text { if } j>m,\end{cases}
$$


so that the signed measure $G_{n}-G_{n}^{\prime}=\sum_{j=1}^{m+m^{\prime}} \varpi_{j, n} \delta_{\vartheta}$ has total mass zero.

The discrepancy orders of the $\vartheta_{j, n}$ 's. We shall first classify the differences between the $\vartheta_{j, n}$ 's in an intrinsic way.

LEMMA 7.1. For a suitable subsequence of $G_{n}-G_{n}^{\prime}$, there is a finite number S of "scaling" sequences

$$
0 \equiv \varepsilon_{0}(n)<\varepsilon_{1}(n)<\cdots<\varepsilon_{S}(n) \equiv 1 \quad \text { with } \varepsilon_{S}(n)=o\left(\varepsilon_{s+1}(n)\right),
$$

such that for all $j, j^{\prime} \in \llbracket 1, m+m^{\prime} \rrbracket$ there is a unique $s\left(j, j^{\prime}\right) \in \llbracket 0, S \rrbracket$ satisfying

$$
\left|\vartheta_{j, n}-\vartheta_{j^{\prime}, n}\right| \asymp \varepsilon_{s\left(j, j^{\prime}\right)}(n) .
$$

The proof is given in Heinrich and Kahn (2018), Appendix C. It follows from the definition of $s\left(j, j^{\prime}\right)$ that $s\left(j, j^{\prime}\right) \leq \max \left(s\left(j, j^{\prime \prime}\right), s\left(j^{\prime}, j^{\prime \prime}\right)\right)$, and thus $s(\cdot, \cdot)$ defines an ultrametric on $\llbracket 1, m+m^{\prime} \rrbracket$. The ultrametric makes any two balls either included one into the other or disjoint, and allows us to build a coarse-graining tree.

DEFINITION 7.2. The coarse-graining tree $\mathcal{T}$ is the collection of distinct balls $J=\{s(\cdot, j) \leq s\}$, called nodes, when $j$ ranges over $\llbracket 1, m+m^{\prime} \rrbracket$ and $s$ over $\llbracket 0, S \rrbracket$. Moreover:

- The root of $\mathcal{T}$ is $J_{r}=\llbracket 1, m+m^{\prime} \rrbracket$,

- The parent $J^{\uparrow}$ of a node $J$ is defined by

$$
\left(J \subset I \subsetneq J^{\uparrow}, I \in \mathcal{T}\right) \quad \Longrightarrow \quad I=J,
$$

- The set of children of a node $J$ is $\operatorname{Child}(J)=\left\{I \in \mathcal{T}: I^{\uparrow}=J\right\}$,

- The set of descendants of a node $J$ is $\operatorname{Desc}(J)=\left\{I \in \mathcal{T}: I^{\uparrow} \subset J\right\}$,

- The diameter of a node $J$ is $s(J)=\max _{j, j^{\prime} \in J} s\left(j, j^{\prime}\right)$.

Let us show how the tree $\mathcal{T}$ looks like with a partial representation (Figure 1).

Note that the ends are not necessarily singletons since the ultrametric $s(\cdot, \cdot)$ does not separate points. Note also that $j$ and $j^{\prime}$ are in different children $K$ and $K^{\prime}$ so that $\left|\vartheta_{j, n}-\vartheta_{j^{\prime}, n}\right|$ is actually exactly of order $\varepsilon_{S(J)}(n)$.

The Wassertsein distances $W_{q}\left(G_{n}, G_{n}^{\prime}\right)$ through the coarse graining tree $\mathcal{T}$. In what follows, $n$ is skipped in the $\vartheta_{j}$ 's, $\varpi_{j}$ 's and $\varepsilon_{s}$ 's. Set for short

$$
\varpi_{J}=\sum_{j \in J} \varpi_{j} \quad \text { and } \quad \varepsilon_{J}=\varepsilon_{s}(J) .
$$

LEMMA 7.3. For any $q \geq 1$, we have

$$
W_{q}\left(G_{n}, G_{n}^{\prime}\right)^{q} \asymp \max _{J \in \operatorname{Desc}\left(J_{r}\right)}\left|\varpi_{J}\right| \varepsilon_{J^{\uparrow}}^{q} .
$$




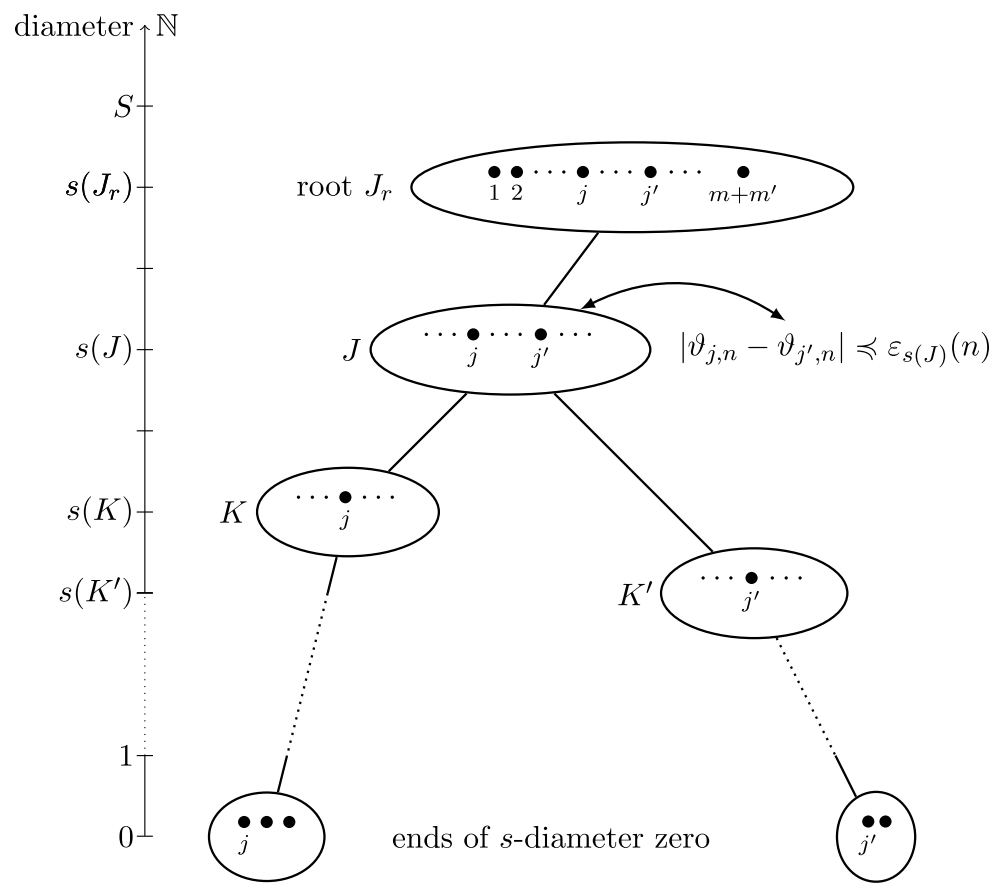

FIG. 1. The coarse-graining tree $\mathcal{T}$.

Proof. Consider any coupling $\Pi$ between $G_{n}$ and $G_{n}^{\prime}$ and set

$$
\begin{aligned}
\Pi\left(J, J^{\prime}\right) & =\Pi\left(\left\{\vartheta_{j}\right\}_{j \in J \cap \llbracket 1, m \rrbracket} \times\left\{\vartheta_{j}\right\}_{j \in J^{\prime} \cap \llbracket m+1, m+m^{\prime} \rrbracket}\right), \\
w_{q}\left(J, J^{\prime}\right) & =\sum_{\left(j, j^{\prime}\right) \in J \times J^{\prime}} \Pi\left(\{j\},\left\{j^{\prime}\right\}\right)\left|\vartheta_{j}-\vartheta_{j^{\prime}}\right|^{q} .
\end{aligned}
$$

Set also $\pi_{J}=\sum_{j \in J \cap \llbracket 1, m \rrbracket} \varpi_{j}$ and $\pi_{J}^{\prime}=-\sum_{j \in J \cap \llbracket m+1, m+m^{\prime} \rrbracket} \varpi_{j}$. These define the marginal distributions of $\Pi$ and we have $\Pi(J, J) \leq \pi_{J} \wedge \pi_{J}^{\prime}$. Note also that $\left|\varpi_{J}\right|=\pi_{J} \vee \pi_{J}^{\prime}-\pi_{J} \wedge \pi_{J}^{\prime}$. With $J^{c}=J_{r} \backslash J$, this gives

$$
\Pi\left(J, J^{c}\right) \vee \Pi\left(J^{c}, J\right) \geq\left|\varpi_{J}\right| .
$$

Notice moreover that if $\left(j, j^{\prime}\right) \in J \times J^{c}$, then $\left|\vartheta_{j}-\vartheta_{j^{\prime}}\right| \succcurlyeq \varepsilon_{J^{\uparrow}}$. Hence, the lower bound of Lemma 7.3 follows from

$$
w_{q}\left(J_{r}, J_{r}\right) \geq w_{q}\left(J, J^{c}\right)+w_{q}\left(J^{c}, J\right) \succcurlyeq\left|\varpi_{J}\right| \varepsilon_{J \uparrow}^{q} .
$$

Conversely, for the upper bound, we show recursively that for all node $J$,

$$
w_{q}(J, J) \preccurlyeq \max _{K \in \operatorname{Desc}(J)}\left|\varpi_{K}\right| \varepsilon_{K \uparrow}^{q} .
$$


This is obviously true if $J$ is an end node, with the value of zero. Assume that (26) holds for children $K$ of a given node $J$. We may develop $J$ on its children:

$$
w_{q}(J, J)=\sum_{K \in \operatorname{Child}(J)}\left[w_{q}(K, K)+\sum_{\substack{K^{\prime} \in \operatorname{Child}(J) \\ K^{\prime} \neq K}} w_{q}\left(K, K^{\prime}\right)\right] .
$$

Furthermore, we get $w_{q}\left(K, K^{\prime}\right) \preccurlyeq \Pi\left(K, K^{\prime}\right) \varepsilon_{J}^{q}$ from (25) and

$$
\Pi\left(K, K^{\prime}\right) \leq \Pi\left(K, K^{c}\right) \leq \pi_{K}-\Pi(K, K),
$$

and if the coupling $\Pi$ is chosen [see Heinrich and Kahn (2018), Lemma B.2, for a construction] such that $\Pi(K, K)=\pi_{K} \wedge \pi_{K}^{\prime}$ for all node $K$, then it follows that

$$
\Pi\left(K, K^{\prime}\right) \leq\left|\varpi_{K}\right|
$$

and thus

$$
w_{q}(J, J) \preccurlyeq \sum_{K \in \operatorname{Child}(J)}\left[w_{q}(K, K)+\left|\varpi_{K}\right| \varepsilon_{J}^{q}\right] .
$$

The recurrence hypothesis on children $K$ yields then (26).

Expanding $F\left(x, G_{n}\right)-F\left(x, G_{n}^{\prime}\right)$ through the coarse graining tree. The dependence on $n$ is skipped in the following notation. Consider the additive setfunction $J \mapsto F(x, J)=\sum_{j \in J} \varpi_{j} F\left(x, \vartheta_{j}\right)$ and note that $F\left(x, J_{r}\right)$ is equal to $F\left(x, G_{n}\right)-F\left(x, G_{n}^{\prime}\right)$.

LEMMA 7.4. Choose $\vartheta_{J}$ in $\left\{\vartheta_{j}: j \in J\right\}$ for each node $J$ of $\mathcal{T}$.

There are a vector $a_{J}=\left(a_{J}(p)\right)_{0 \leq p \leq 2 \mathrm{~m}}$ and a remainder $R(x, J)$ such that

$$
F(x, J)=\sum_{p=0}^{2 \mathbf{m}} a_{J}(p) \varepsilon_{J}^{p} F^{(p)}\left(x, \vartheta_{J}\right)+R(x, J),
$$

where:

(a) $a_{J}(0)=\varpi_{J}$ and $\left\|a_{J}\right\| \preccurlyeq 1$,

(b) There is an integer $p_{J}<|J|$ such that $\left\|a_{J}\right\| \asymp\left|a_{J}\left(p_{J}\right)\right|$,

(c) The norm $\left\|a_{J}\right\|$ is bounded from below by a quantity linked to $W_{q}$ :

$$
\left\|a_{J}\right\| \succcurlyeq \max _{K \in \operatorname{Desc}(J)}\left[\left|\varpi_{K}\right|\left(\frac{\varepsilon_{K} \uparrow}{\varepsilon_{J}}\right)^{|J|-1}\right],
$$

(d) $R(x, J)=o\left(\left\|a_{J}\right\| \varepsilon_{J}^{2 m}\right)$ uniformly in $x$.

As a remark, the lower bound on $F\left(x, J_{r}\right)$ will stem from points (c) and (d). Points (a) and (b) are mainly there for transmitting recurrence hypotheses. They control the size of $F(x, J)$, together with point (c). The behaviour of $F(x, J)$ only 
depends on the first $|J|$ terms in the sum. However, the sum goes to $2 \mathbf{m}$ so that it is useful when $J=J_{r}$.

PROOF. The proof uses Taylor expansions at $\theta_{K}$ for a given generation of children $K$ together with separation and order properties of the coarse-graining tree $\mathcal{T}$. Recall notation (24).

If $K$ is an end of the tree $\mathcal{T}$, then all the $\theta_{j}$ for $j \in K$ are equal, and $F(x, K)=$ $\varpi_{K} F\left(x, \theta_{K}\right)$. Choose $a_{K}(p)=\varpi_{K} \mathbf{1}_{\{p=0\}}$ and $R(x, K)=0$ so that the equality (27) holds for the end node $K$ with all the desired estimates (a), (b), (c) and (d).

Assume now that $J$ has children $K$, each of them satisfying (27) with all the estimates (a), (b), (c) and (d):

$$
F(x, K)=\sum_{\ell=0}^{2 \mathbf{m}} a_{K}(\ell) \varepsilon_{K}^{\ell} F^{(\ell)}\left(x, \vartheta_{K}\right)+R(x, K) .
$$

We want to transmit (28) and the estimates to the parent $J$. Suppose without loss of generality that $\vartheta_{J} \leq \vartheta_{K}$ and apply Taylor's formula with remainder to $F^{(\ell)}\left(x, \vartheta_{K}\right)$ at $\vartheta_{J}$ for all $\ell \in \llbracket 0,2 \mathbf{m} \rrbracket$ :

$$
\begin{gathered}
F^{(\ell)}\left(x, \vartheta_{K}\right)-\sum_{p=\ell}^{2 \mathbf{m}-1} \frac{\left(\vartheta_{K}-\vartheta_{J}\right)^{p-\ell}}{(p-\ell) !} F^{(p)}\left(x, \vartheta_{J}\right) \\
=\int_{\vartheta_{J}}^{\vartheta_{K}} \frac{\left(\vartheta_{K}-\theta\right)^{2 \mathbf{m}-1-\ell}}{(2 \mathbf{m}-1-\ell) !} F^{(2 m)}(x, \theta) \mathrm{d} \theta .
\end{gathered}
$$

Subtract the term $\frac{\left(\vartheta_{K}-\vartheta_{J}\right)^{2 \mathbf{m}-\ell}}{(2 \mathbf{m}-\ell) !} F^{(2 \mathbf{m})}\left(x, \vartheta_{J}\right)$ from either side so that

$$
\begin{aligned}
F^{(\ell)}\left(x, \vartheta_{K}\right)-\sum_{p=\ell}^{2 \mathbf{m}} \frac{\left(\vartheta_{K}-\vartheta_{J}\right)^{p-\ell}}{(p-\ell) !} F^{(p)}\left(x, \vartheta_{J}\right) \\
\quad=\int_{\vartheta_{J}}^{\vartheta_{K}} \frac{\left(\vartheta_{K}-\theta\right)^{2 \mathbf{m}-1-\ell}}{(2 \mathbf{m}-1-\ell) !}\left[F^{(2 \mathbf{m})}(x, \theta)-F^{(2 \mathbf{m})}\left(x, \vartheta_{J}\right)\right] \mathrm{d} \theta \\
=\left(\vartheta_{K}-\vartheta_{J}\right)^{2 \mathbf{m}-\ell} O\left(\sup _{\theta \in\left[\vartheta_{J}, \vartheta_{K}\right]}\left|F^{(2 \mathbf{m})}(x, \theta)-F^{(2 \mathbf{m})}\left(x, \vartheta_{J}\right)\right|\right) .
\end{aligned}
$$

The modulus of continuity of $F^{(2 \mathbf{m})}(x, \cdot)$ from $\mathrm{B}(2 \mathbf{m})$ then yields

$$
F^{(\ell)}\left(x, \vartheta_{K}\right)=\sum_{p=\ell}^{2 \mathbf{m}} \frac{\left(\vartheta_{K}-\vartheta_{J}\right)^{p-\ell}}{(p-\ell) !} F^{(p)}\left(x, \vartheta_{J}\right)+o\left(\left(\vartheta_{K}-\vartheta_{J}\right)^{2 \mathbf{m}-\ell}\right) .
$$

The normalised discrepancies $\phi_{K}:=\left(\vartheta_{K}-\vartheta_{J}\right) / \varepsilon_{J}$ for $K \in \operatorname{Child}(J)$ are by definition at most of order 1 so that

$$
F^{(\ell)}\left(x, \vartheta_{K}\right)=\sum_{p=\ell}^{2 \mathbf{m}} \varepsilon_{J}^{p-\ell} \frac{\phi_{K}^{p-\ell}}{(p-\ell) !} F^{(p)}\left(x, \vartheta_{J}\right)+\varepsilon_{J}^{2 \mathbf{m}-\ell} o(1) .
$$


Substitute in (28) and change the order of summation:

$$
\begin{aligned}
F(x, K)= & \sum_{p=0}^{2 \mathbf{m}}\left[\sum_{\ell=0}^{p} a_{K}(\ell)\left(\frac{\varepsilon_{K}}{\varepsilon_{J}}\right)^{\ell} \frac{\phi_{K}^{p-\ell}}{(p-\ell) !}\right] \varepsilon_{J}^{p} F^{(p)}\left(x, \vartheta_{J}\right) \\
& +R(x, K)+\varepsilon_{J}^{2 \mathbf{m}} \max _{0 \leq \ell \leq 2 \mathbf{m}}\left|a_{K}(\ell)\left(\frac{\varepsilon_{K}}{\varepsilon_{J}}\right)^{\ell}\right| o(1) .
\end{aligned}
$$

Add up over the children $K$ of $J$ to obtain (27) for $J$, that is,

$$
F(x, J)=\sum_{p=0}^{2 m} a_{J}(p) \varepsilon_{J}^{p} F^{(p)}\left(x, \vartheta_{J}\right)+R(x, J),
$$

with $a_{J}(p)=\sum_{K \in \operatorname{Child}(J)} \sum_{\ell=0}^{p} a_{K}(\ell)\left(\frac{\varepsilon_{K}}{\varepsilon_{J}}\right)^{\ell} \frac{\phi_{K}^{p-\ell}}{(p-\ell) !}$ and

$$
R(x, J)=\sum_{K \in \operatorname{Child}(J)}\left[R(x, K)+\varepsilon_{J}^{2 \mathbf{m}} \max _{0 \leq \ell \leq 2 \mathbf{m}}\left|a_{K}(\ell)\left(\frac{\varepsilon_{K}}{\varepsilon_{J}}\right)^{\ell}\right| o(1)\right] .
$$

We have now to prove the estimates (a), (b), (c) and (d) for the defined coefficients $a_{J}(p)$ and remainder $R(x, J)$. Keep in mind that these estimates are assumed to be true for the children $K$ and set for short

$$
M_{p, K}:=\max _{0 \leq \ell \leq p}\left|a_{K}(\ell)\left(\frac{\varepsilon_{K}}{\varepsilon_{J}}\right)^{\ell}\right| \text {. }
$$

Proof of (a) for $J$. It is immediate from the definition of $a_{J}(p)$ that

$$
a_{J}(0)=\sum_{K \in \operatorname{Child}(J)} a_{K}(0)=\sum_{K \in \operatorname{Child}(J)} \varpi_{K}=\varpi_{J},
$$

and, using (a) for $K$ together with $\varepsilon_{K} \leq \varepsilon_{J}$, we get

$$
\left|a_{J}(p)\right| \preccurlyeq \max _{K \in \operatorname{Child}(J)} M_{p, K} \preccurlyeq 1 \text {. }
$$

Proof of (b) for J. It's enough to establish

$$
\max _{|J| \leq p \leq 2 \mathbf{m}}\left|a_{J}(p)\right| \preccurlyeq \max _{K \in \operatorname{Child}(J)} M_{|K|-1, K} \asymp \max _{0 \leq p<|J|}\left|a_{J}(p)\right| .
$$

To prove the left-hand side of (31), note from (30) that $\left|a_{J}(p)\right| \preccurlyeq$ $\max _{K \in \operatorname{Child}(J)} M_{p, K}$. Moreover, for all $p \geq|K|$,

$$
M_{p, K} \leq M_{|K|-1, K}+\max _{|K| \leq \ell \leq p}\left|a_{K}(\ell)\left(\frac{\varepsilon_{K}}{\varepsilon_{J}}\right)^{\ell}\right| \leq M_{|K|-1, K}+\left\|a_{K}\right\|\left(\frac{\varepsilon_{K}}{\varepsilon_{J}}\right)^{|K|}
$$

and we have $\left\|a_{K}\right\| \asymp \max _{0 \leq \ell<|K|}\left|a_{K}(\ell)\right|$ by (b) so that, even for $p<|K|$,

$$
M_{p, K} \preccurlyeq\left(1+\frac{\varepsilon_{K}}{\varepsilon_{J}}\right) M_{|K|-1, K} \preccurlyeq M_{|K|-1, K} .
$$


Taking the supremum over $K \in \operatorname{Child}(J)$ and over $p$ give the left-hand side of (31).

To prove the right-hand side of (31), write $a_{J}(p)=a_{J}^{(1)}(p)+a_{J}^{(2)}(p)$ with

$$
\begin{aligned}
& a_{J}^{(1)}(p)=\sum_{K \in \operatorname{Child}(J)} \sum_{\ell=0}^{|K|-1} a_{K}(\ell)\left(\frac{\varepsilon_{K}}{\varepsilon_{J}}\right)^{\ell} \frac{\phi_{K}^{p-\ell}}{(p-\ell) !} \mathbf{1}_{p \geq \ell}, \\
& a_{J}^{(2)}(p)=\sum_{K \in \operatorname{Child}(J)} \sum_{\ell=|K|}^{p} a_{K}(\ell)\left(\frac{\varepsilon_{K}}{\varepsilon_{J}}\right)^{\ell} \frac{\phi_{K}^{p-\ell}}{(p-\ell) !} \mathbf{1}_{p \geq \ell .}
\end{aligned}
$$

Note that $\left\{\phi_{K}\right\}_{K \in \operatorname{Child}(J)}$ is $\varepsilon$-separated since $\left|\phi_{K}-\phi_{K^{\prime}}\right|=\left|\vartheta_{K}-\vartheta_{K^{\prime}}\right| / \varepsilon_{J} \asymp 1$ for $K \neq K^{\prime}$. Set $\lambda_{K, \ell}=a_{K}(\ell)\left(\varepsilon_{K} / \varepsilon_{J}\right)^{\ell}$ and apply Corollary D.2 of Heinrich and Kahn (2018) to $A\left(\left\{\phi_{K}\right\}_{K \in \operatorname{Child}(J)}\right)$ and $\Lambda=\left(\lambda_{K, \ell}\right)_{K \in \operatorname{Child}(J), 0 \leq \ell<|K|}$ :

$$
\max _{0 \leq p<|J|}\left|a_{J}^{(1)}(p)\right| \asymp \max _{\substack{K \in \operatorname{Child}(J) \\ 0 \leq \ell<|K|}}\left|a_{K}(\ell)\left(\frac{\varepsilon_{K}}{\varepsilon_{J}}\right)^{\ell}\right|=\max _{K \in \operatorname{Child}(J)} M_{|K|-1, K},
$$

which is the right-hand side of (31) with $a_{J}^{(1)}(\cdot)$ instead of $a_{J}(\cdot)$.

We show now that the $\left|a_{J}^{(2)}(p)\right|$ are in fact negligible so that the right-hand side of (31) will follows. Indeed, easy bounds on $a_{J}^{(2)}(p)$ yield

$$
\max _{0 \leq p<|J|}\left|a_{J}^{(2)}(p)\right| \preccurlyeq \max _{K \in \operatorname{Child}(J)}\left[\left\|a_{K}\right\|\left(\frac{\varepsilon_{K}}{\varepsilon_{J}}\right)^{|K|}\right],
$$

whereas, as a by-product of (33), using $\left\|a_{K}\right\| \asymp \max _{0 \leq \ell<|K|}\left|a_{K}(\ell)\right|$,

$$
\max _{0 \leq p<|J|}\left|a_{J}^{(1)}(p)\right| \succcurlyeq \max _{K \in \operatorname{Child}(J)}\left[\left\|a_{K}\right\|\left(\frac{\varepsilon_{K}}{\varepsilon_{J}}\right)^{|K|-1}\right] .
$$

Proof of (c) for $J$. From the right-hand side of (31), and (a) for $K$, we deduce

$$
\left\|a_{J}\right\| \succcurlyeq \max _{K \in \operatorname{Child}(J)}\left[\left\|a_{K}\right\|\left(\frac{\varepsilon_{K}}{\varepsilon_{J}}\right)^{|K|-1}\right] \vee \max _{K \in \operatorname{Child}(J)}\left|\varpi_{K}\right| .
$$

Here, we used $M_{|K|-1, K} \geq M_{0, K}=\left|\varpi_{K}\right|$. Combining (34) with (c) for children $K$ gives

$$
\left\|a_{J}\right\| \succcurlyeq \max _{K \in \operatorname{Child}(J)} \max _{F \in \operatorname{Desc}(K)}\left[\left|\pi_{F}\right|\left(\frac{\varepsilon_{F} \uparrow}{\varepsilon_{J}}\right)^{|K|-1}\right] \vee \max _{K \in \operatorname{Child}(J)}\left|\varpi_{K}\right| .
$$

Now, bound the exponent $|K|$ by $|J|$ to derive (c) for $J$.

Proof of (d) for $J$. Split (29) as $R(x, J)=R^{(1)}(x, J)+R^{(2)}(x, J)$ with

$$
\begin{aligned}
& R^{(1)}(x, J)=\sum_{K \in \operatorname{Child}(J)} R(x, K), \\
& R^{(2)}(x, J)=\varepsilon_{J}^{2 \mathbf{m}} \sum_{K \in \operatorname{Child}(J)} M_{2 \mathbf{m}, K} o(1) .
\end{aligned}
$$


Note that (31) and (32) give $\max _{K \in \operatorname{Child}(J)} M_{2 \mathbf{m}, K} \asymp\left\|a_{J}\right\|$; moreover,

$$
\left\|R^{(1)}(\cdot, J)\right\|_{\infty} \preccurlyeq \max _{K \in \operatorname{Child}(J)}\left[o\left(\left\|a_{K}\right\| \varepsilon_{K}^{2 \mathbf{m}}\right)\right]
$$

by assumption (d) for $K$, so that by triangle inequality,

$$
\|R(\cdot, J)\|_{\infty} \preccurlyeq \varepsilon_{J}^{2 \mathbf{m}}\left\{\max _{K \in \operatorname{Child}(J)}\left[o\left(\left\|a_{K}\right\|\left(\frac{\varepsilon_{K}}{\varepsilon_{J}}\right)^{2 \mathbf{m}}\right)\right]+\left\|a_{J}\right\| o(1)\right\} .
$$

By (34), $\left\|a_{J}\right\|$ dominates $\left\|a_{K}\right\|\left(\varepsilon_{K} / \varepsilon_{J}\right)^{2 \mathbf{m}}$, and thus (d) follows for $J$.

Concluding the proof of (23). We shall show that

$$
\left\|F\left(\cdot, G_{n}\right)-F\left(\cdot, G_{n}^{\prime}\right)\right\|_{\infty} \succcurlyeq \max _{J \in \operatorname{Desc}\left(J_{r}\right)}\left|\varpi_{J}\right| \varepsilon_{J \uparrow}^{2 d_{0}+1} .
$$

Recall that $F\left(x, G_{n}\right)-F\left(x, G_{n}^{\prime}\right)=F\left(x, J_{r}\right)$ and distinguish two cases:

Case $\varepsilon_{J_{r}} \rightarrow 0$. All the $\vartheta_{j, n}$ 's converge to a single support point of $G_{0}$ so that $m_{0}=1$. Apply directly Lemma 7.4 to the root node $J:=J_{r}$ :

$$
F(x, J)=\sum_{p=0}^{2 \mathbf{m}} a_{J}(p) \varepsilon_{J}^{p} F^{(p)}\left(x, \vartheta_{J}\right)+R(x, J),
$$

so that by the triangle's inequality, Proposition 2.3 and (d) for $J$,

$$
\|F(\cdot, J)\|_{\infty} \succcurlyeq \max _{0 \leq p \leq 2 \mathbf{m}}\left|a_{J}(p) \varepsilon_{J}^{p}\right|-o\left(\left\|a_{J}\right\| \varepsilon_{J}^{2 \mathbf{m}}\right) .
$$

By (b), the optimal $p$ is at most $|J|-1$, and since $|J| \leq 2 \mathbf{m}$, we get

$$
\|F(\cdot, J)\|_{\infty} \succcurlyeq\left\|a_{J}\right\| \varepsilon_{J}^{|J|-1} \text {. }
$$

Now, the estimate (c) for $J$ yields further

$$
\|F(\cdot, J)\|_{\infty} \succcurlyeq \max _{K \in \operatorname{Desc}(J)}\left|\varpi_{K}\right| \varepsilon_{K^{\uparrow}}^{2 \mathbf{m}-1} .
$$

But this estimate is nothing else than (35) since $m_{0}=\mathbf{m}-d_{0}$ is one.

Case $\varepsilon_{J_{r}} \equiv 1$. This case means either there are more than one support point in the limit $G_{0}\left(m_{0}>1\right)$ or there is only one support point for $G_{0}$ but with possible sequences $\theta_{j, n}$ converging to other points (vanishing weights $\varpi_{j, n}$ may exist).

Here, all the $\varepsilon_{J_{r}}^{p}$,s are of the same order (actually identical), so the scheme of the case $\varepsilon_{J_{r}} \rightarrow 0$ does not work. It works however for the children $J$ of $J_{r}$ :

$$
\begin{aligned}
F\left(x, J_{r}\right) & =\sum_{J \in \operatorname{Child}\left(J_{r}\right)} F(x, J) \\
& =\sum_{J \in \operatorname{Child}\left(J_{r}\right)} \sum_{p=0}^{2 \mathbf{m}} a_{J}(p) \varepsilon_{J}^{p} F^{(p)}\left(x, \vartheta_{J}\right)+\sum_{J \in \operatorname{Child}\left(J_{r}\right)} R(x, J),
\end{aligned}
$$


so that by the triangle's inequality, Proposition 2.3 and (d) for $J$,

$$
\left\|F\left(\cdot, J_{r}\right)\right\|_{\infty} \succcurlyeq \max _{J \in \operatorname{Child}\left(J_{r}\right)} \max _{0 \leq p \leq 2 \mathbf{m}}\left|a_{J}(p) \varepsilon_{J}^{p}\right|-\max _{J \in \operatorname{Child}\left(J_{r}\right)} o\left(\left\|a_{J}\right\| \varepsilon_{J}^{2 \mathbf{m}}\right) .
$$

The optimal $p$ is at most $|J|-1$ by (b), so that $\left\|F\left(\cdot, J_{r}\right)\right\|_{\infty}$ dominates $\max _{J \in \operatorname{Child}\left(J_{r}\right)}\left\|a_{J}\right\| \varepsilon_{J}^{|J|-1}$, whereas for $p=0$ we get that $\left\|F\left(\cdot, J_{r}\right)\right\|_{\infty}$ dominates $\max _{J \in \operatorname{Child}\left(J_{r}\right)}\left|\varpi_{J}\right|$, by (a). Together with $\varepsilon_{J \uparrow} \equiv 1$ and (c) for $J$, we obtain

$$
\left\|F\left(\cdot, J_{r}\right)\right\|_{\infty} \succcurlyeq \max _{J \in \operatorname{Child}\left(J_{r}\right)} \max _{K \in \operatorname{Desc}(J) \cup\{J\}}\left|\varpi_{K}\right| \varepsilon_{K^{\uparrow}}^{|J|-1},
$$

which is nothing else than

$$
\left\|F\left(\cdot, G_{n}\right)-F\left(\cdot, G_{n}^{\prime}\right)\right\|_{\infty} \succcurlyeq \max _{J \in \operatorname{Desc}\left(J_{r}\right)}\left|\varpi_{J}\right| \varepsilon_{J \uparrow}^{|J|-1} .
$$

Now, note that a descendant $J$ of $J_{r}$ of maximal cardinality must be a child of $J_{r}$, call it $J_{\star}$. Since $G_{n}$ and $G_{n}^{\prime}$ converge to $G_{0} \in \mathcal{G}_{m_{0}}$, the root $J_{r}$ has at least $m_{0}$ children, each of them containing at least two points. Thus, we have

$$
\left|J_{r}\right| \geq\left|J_{\star}\right|+2\left(m_{0}-1\right) .
$$

Since $\left|J_{r}\right|$ is at most $2 \mathbf{m}$, we deduce further that $\left|J_{\star}\right| \leq 2 \mathbf{m}-2 m_{0}+2$. From (36), we finally arrive at (35), exactly as in the case $\varepsilon_{J_{r}} \rightarrow 0$.

Now, recall that $q=2 \mathbf{m}-2 m_{0}+1$. Lemma 7.3 together with (35) ensure that, whatever the case, $\varepsilon_{J_{r}} \rightarrow 0$ or $\varepsilon_{J_{r}} \equiv 1$,

$$
\left\|F\left(\cdot, G_{n}\right)-F\left(\cdot, G_{n}^{\prime}\right)\right\|_{\infty} \succcurlyeq W_{q}\left(G_{n}, G_{n}^{\prime}\right)^{q},
$$

which is the stated contradiction (23).

7.2. From local to global: How (18) implies (19). We have to show that, under Assumption $\mathrm{B}(2 \mathbf{m})$, for $r=2 \mathbf{m}-1$,

$$
L:=\inf _{G \neq G^{\prime} \in \mathcal{G}_{\leq \mathbf{m}}} \frac{\left\|F(\cdot, G)-F\left(\cdot, G^{\prime}\right)\right\|_{\infty}}{W_{r}\left(G, G^{\prime}\right)^{r}}>0 .
$$

From the definition of $L$, we can select mixing distributions $G_{n}$ and $G_{n}^{\prime}$ in $\mathcal{G}_{\leq \mathbf{m}}$ such that $\left\|F\left(\cdot, G_{n}\right)-F\left(\cdot, G_{n}^{\prime}\right)\right\|_{\infty} / W_{r}\left(G_{n}, G_{n}^{\prime}\right)^{r}$ converges to $L$. Since the set $\mathcal{G}_{\leq \mathbf{m}} \times \mathcal{G}_{\leq \mathbf{m}}$ is compact, we can assume that $\left(G_{n}, G_{n}^{\prime}\right)$ converges to some limit $\left(G_{\infty}, G_{\infty}^{\prime}\right)$. Set $w=W_{r}\left(G_{\infty}, G_{\infty}^{\prime}\right)$.

Case $w>0$. This case does not depend on (18). By identifiability, there is $x_{0} \in$ $\mathbb{R}$ such that $\Delta_{0}:=\left|F\left(x_{0}, G_{\infty}\right)-F\left(x_{0}, G_{\infty}^{\prime}\right)\right|>0$. Then, for all $n$,

$$
\frac{\left\|F\left(\cdot, G_{n}\right)-F\left(\cdot, G_{n}^{\prime}\right)\right\|_{\infty}}{W_{r}\left(G_{n}, G_{n}^{\prime}\right)^{r}} \geq \frac{\left|F\left(x_{0}, G_{n}\right)-F\left(x_{0}, G_{n}^{\prime}\right)\right|}{W_{r}\left(G_{n}, G_{n}^{\prime}\right)^{r}} .
$$

The numerator of the right-hand side of (37) goes to $\Delta_{0}$ by the triangle's inequality and since the function $\theta \mapsto F\left(x_{0}, \theta\right)$ is Lipschitz w.r.t. the metric $W_{1}$ and thus also w.r.t. $W_{r}$. As a consequence, we get $L \geq \Delta_{0} / w^{r}>0$. 
Case $w=0$. Consider (18) with $G_{0}=G_{\infty}$. For $n$ larger than some $n_{0}$, all $W_{q}\left(G_{n}, G_{0}\right)$ and $W_{q}\left(G_{n}^{\prime}, G_{0}\right)$ are less than $\varepsilon$ so that by (18),

$$
\inf _{n \geq n_{0}} \frac{\left\|F\left(\cdot, G_{n}\right)-F\left(\cdot, G_{n}^{\prime}\right)\right\|_{\infty}}{W_{q}\left(G_{n}, G_{n}^{\prime}\right)^{q}}>\delta .
$$

Since we have $W_{q}(\cdot, \cdot)^{q} \operatorname{Diam}(\Theta)^{r-q} \geq W_{r}(\cdot, \cdot)^{r}$ for $r \geq q$, we get

$$
\inf _{n \geq n_{0}} \frac{\left\|F\left(\cdot, G_{n}\right)-F\left(\cdot, G_{n}^{\prime}\right)\right\|_{\infty}}{W_{r}\left(G_{n}, G_{n}^{\prime}\right)^{r}}>\frac{\delta}{\operatorname{Diam}(\Theta)^{r-q}},
$$

which gives $L \geq \delta / \operatorname{Diam}(\Theta)^{r-q}$ in the limit and (19) in that case.

7.3. Completing the proof of Theorem 6.3: The easy cases (20) and (21). For the proof of (20), we can simply make use of Theorem 3.1 of Ho and Nguyen (2015). Alternatively, a detailed proof with our notations is available in the Supplementary Material [Heinrich and Kahn (2018), B.2].

For the proof of (21), we can follow the proof of Chen (1995), Lemma 2, which holds here, because the $\gamma_{j}$ defined in his paper are all nonnegative, and at least one is nonzero.

Acknowledgements. We thank Sébastien Gadat for numerous suggestions that have greatly improved the presentation of the paper, and Élisabeth Gassiat for helpful discussions. We also thank the referees for many remarks.

\section{SUPPLEMENTARY MATERIAL}

Auxiliary results and technical details (DOI: 10.1214/17-AOS1641SUPP; .pdf). This supplemental part gathers some proof details on some assertions given in the paper.

\section{REFERENCES}

Bontemps, D. and GADAT, S. (2014). Bayesian methods for the shape invariant model. Electron. J. Stat. 8 1522-1568. MR3263130

Caillerie, C., Chazal, F., Dedecker, J. and Michel, B. (2013). Deconvolution for the Wasserstein metric and geometric inference. In Geometric Science of Information 561-568. Springer, Berlin.

CHEN, J. H. (1995). Optimal rate of convergence for finite mixture models. Ann. Statist. 23 221-233. MR1331665

Dacunha-Castelle, D. and Gassiat, E. (1997). The estimation of the order of a mixture model. Bernoulli 3 279-299. MR1468306

DEDECKER, J. and MiCHEL, B. (2013). Minimax rates of convergence for Wasserstein deconvolution with supersmooth errors in any dimension. J. Multivariate Anal. 122 278-291.

DEELY, J. J. and KRUSE, R. L. (1968). Construction of sequences estimating the mixing distribution. Ann. Math. Stat. 39 286-288.

Dudley, R. M. (2002). Real Analysis and Probability. Cambridge Studies in Advanced Mathematics 74. Cambridge Univ. Press, Cambridge. MR1932358 
FAN, J. (1991). On the optimal rates of convergence for nonparametric deconvolution problems. Ann. Statist. 19 1257-1272. MR1126324

GASSIAT, E. and VAN HANDEL, R. (2013). Consistent order estimation and minimal penalties. IEEE Trans. Inform. Theory $\mathbf{5 9} 1115-1128$. MR3015722

Genovese, C. R. and WASSERMAN, L. (2000). Rates of convergence for the Gaussian mixture sieve. Ann. Statist. 28 1105-1127. MR1810921

GHOSAL, S. and VAN DER VAART, A. W. (2001). Entropies and rates of convergence for maximum likelihood and Bayes estimation for mixtures of normal densities. Ann. Statist. 29 1233-1263. MR1873329

HÁJEK, J. (1972). Local asymptotic minimax and admissibility in estimation. In Proceedings of the Sixth Berkeley Symposium on Mathematical Statistics and Probability (Univ. California, Berkeley, Calif., 1970/1971), Vol. I: Theory of Statistics 175-194. Univ. California Press, Berkeley, CA.

HeINRICH, P. and KAHN, J. (2018). Supplement to "Strong identifiability and optimal minimax rates for finite mixture estimation." DOI:10.1214/17-AOS1641SUPP.

Ho, N. and NGUYEN, X. (2015). Identifiability and optimal rates of convergence for parameters of multiple types in finite mixtures. Preprint. Available at arXiv:1501.02497.

Ho, N. and NGUYEN, X. (2016a). On strong identifiability and convergence rates of parameter estimation in finite mixtures. Electron. J. Stat. 10 271-307. MR3466183

Ho, N. and NGuYen, X. (2016b). Convergence rates of parameter estimation for some weakly identifiable finite mixtures. Ann. Statist. 44 2726-2755. MR3576559

Holzmann, H., Munk, A. and Stratmann, B. (2004). Identifiability of finite mixtures-with applications to circular distributions. Sankhyā 66 440-449. MR2108200

IsHWARAN, H., JAMES, L. F. and SUn, J. (2001). Bayesian model selection in finite mixtures by marginal density decompositions. J. Amer. Statist. Assoc. 96 1316-1332. MR1946579

Kuhn, M. A., Feigelson, E. D., Getman, K. V., Baddeley, A. J., Broos, P. S., Sills, A., Bate, M. R., Povich, M. S., Luhman, K. L., Busk, H. A. et al. (2014). The spatial structure of young stellar clusters. I. Subclusters. The Astrophysical Journal 787107.

LE CAM, L. (1960). Locally asymptotically normal families of distributions. Certain approximations to families of distributions and their use in the theory of estimation and testing hypotheses. Univ. California Publ. Statist. 3 37-98. MR0126903

Le CAM, L. (1986). Asymptotic Methods in Statistical Decision Theory. Springer, New York.

Lindsay, B. G. (1989). Moment matrices: Applications in mixtures. Ann. Statist. 17 722-740. MR0994263

LIU, M. and HANCOCK, G. R. (2014). Unrestricted mixture models for class identification in growth mixture modeling. Educational and Psychological Measurement 74 557-584.

MARTIN, R. (2012). Convergence rate for predictive recursion estimation of finite mixtures. Statist. Probab. Lett. 82 378-384.

MASSART, P. (1990). The tight constant in the Dvoretzky-Kiefer-Wolfowitz inequality. Ann. Probab. 18 1269-1283. MR1062069

McLachlan, G. and Peel, D. (2000). Finite Mixture Models. Wiley, New York.

NGUYeN, X. (2013). Convergence of latent mixing measures in finite and infinite mixture models. Ann. Statist. 41 370-400. MR3059422

Pearson, K. (1894). Contributions to the theory of mathematical evolution. Philos. Trans. R. Soc. Lond. Ser. A Math. Phys. Eng. Sci. 185 71-110.

Rousseau, J. and Mengersen, K. (2011). Asymptotic behaviour of the posterior distribution in overfitted mixture models. J. R. Stat. Soc. Ser. B. Stat. Methodol. 73 689-710.

TeH, Y. W. (2010). Dirichlet process. In Encyclopedia of Machine Learning (C. Sammut and G. Webb, eds.) 280-287. Springer, New York.

Titterington, D. M., Smith, A. F. M. and Makov, U. E. (1985). Statistical Analysis of Finite Mixture Distributions. Wiley, Chichester. 
VAN DE GEER, S. (1996). Rates of convergence for the maximum likelihood estimator in mixture models. J. Nonparametr. Stat. 6 293-310.

VAn Der VAarT, A. W. (1998). Asymptotic Statistics. Cambridge Series in Statistical and Probabilistic Mathematics 3. Cambridge Univ. Press, Cambridge.

YANG, Y. (2005). Can the strengths of AIC and BIC be shared? A conflict between model indentification and regression estimation. Biometrika 92 937-950. MR2234196

Zhu, H.-T. and ZHANG, H. (2004). Hypothesis testing in mixture regression models. J. R. Stat. Soc. Ser. B. Stat. Methodol. 66 3-16. MR2035755

ZHU, H. and ZHANG, H. (2006). Asymptotics for estimation and testing procedures under loss of identifiability. J. Multivariate Anal. 97 19-45. MR2208842

Laboratoire Paul Painlevé BÂt. M2

Cité SCIENTIFIQUE

UNIVERSITÉ LILLE 1

VILLENEUVE D'ASCQ

69655 CEDEX

FRANCE

E-MAIL: philippe.heinrich@math.univ-lille1.fr
UNIVERSITÉ PAUL SABATIER

InSTITUT DE MATHÉMATIQUES DE TOULOUSE 118 RoUTE DE NARBONNE

F-31062 TOULOUSE CEDEX 9

FRANCE

E-MAIL: jonas.kahn@math.univ-toulouse.fr 